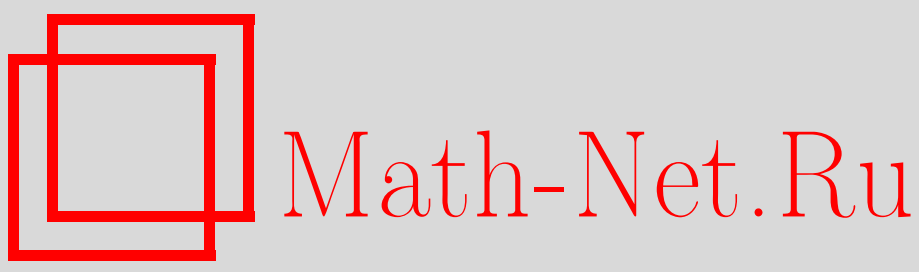

Е. А. Калита, Сингулярные решения нелинейных эллиптических систем высокого порядка, Матем. сб., 2004, том 195, номер 11, 63-94

DOI: https://doi.org/10.4213/sm859

Использование Общероссийского математического портала Math-Net.Ru подразумевает, что вы прочитали и согласны с пользовательским соглашением http://www . mathnet.ru/rus/agreement

Параметры загрузки:

IP: 34.229 .108 .108

26 апреля 2023 г., $07: 22: 53$ 
УДК 517.956

\author{
Е. А. Калита
}

\title{
Сингулярные решения нелинейных Эллиптических систем высокого порядка
}

\begin{abstract}
Изучается поведение решений нелинейных эллиптических систем в окрестности особой точки, конечной или бесконечной. Устанавливается, что если порядок особенности попадает в некоторьй интервал, зависящий от модуля эллиптичности системы, то он совпадает с порядком особенности одного из сингулярных решений полилапласиана. Для таких решений получены точные двусторонние оценки энергии вблизи особой точки. Рассмотрены глобальные решения, для которых из оценок сверху получены оценки энергии снизу. Для уравнений и систем второго порядка построены контрпримеры, показывающие, что интервал правильного поведения порядка особенности определен точно.

Библиографиия: 10 названий.
\end{abstract}

Поведение решений эллиптических уравнений и систем в окрестности особой точки изучалось во многих работах. Но в большинстве случаев рассматриваются линейные системы или системы с линейной главной частью. Укажем, например, работу [1], где для эллиптических систем с постоянными коэффициентами получено асимптотическое разложение решения в окрестности бесконечности. Из собственно нелинейной теории отметим работу [2], где исследованы сингулярности решений нелинейных эллиптических уравнений второго порядка дивергентного вида.

Изучение качественных свойств решений нелинейных эллиптических уравнений и систем высокого порядка ограничено из-за недостаточности методов. В теории регулярности решений плодотворной оказалась идея Кордеса [3] об использовании интегральных оценок со степенным весом, где показатель веса ограничен величиной, определяемой модулем эллиптичности уравнения. В нашей работе [4] эта идея была применена при изучении особых точек решений.

Настоящая работа развивает результаты и методы работы [4]. В $\S 1$ рассматривается поведение решений в окрестности особой точки. Показано, что если порядок особенности (в смысле скорости роста/убывания энергии) попадает в некоторый интервал, определяемый модулем эллиптичности системы, то он совпадает с порядком особенности одного из сингулярных решений полилапласиана. В отличие от [4], где оценки роста энергии в этом результате установлены с точностью до $R^{\varepsilon}, R$ - расстояние до особой точки, здесь получены двусторонние оценки с точностью до мультипликативной константы. Такие же оценки получены на концах интервала, где могут появляться решения с “неправильным" порядком особенности. Оценки величины интервала несколько улучшены в сравнении с [4]. В 22 устанавливаются интегральные оценки решения с бистепенным весом (весом типа $\left.\min \left\{r^{a} ; r^{b}\right\}\right)$, на основе которых доказываются теоремы $\S 1$. В $\S 3$ рассмотрены глобальные решения, имеющие не более одной конечной особой точки в $\mathbb{R}^{n}$. Для таких 
решений показано, что из оценок энергии сверху следуют соответствующие оценки энергии снизу. Типичньй результат следующий: если вблизи конечной (или бесконечной) особой точки нетривиальное решение допускает оценку энергии сверху такую, как некоторое сингулярное решение полилапласиана, то вблизи бесконечной (или конечной) особой точки для него имеет место оценка энергии снизу такая, как для того же решения полилапласиана. Аналогичные результаты получены для порядков особенностей, соответствующих концам интервала. В $\S 4$ построены примеры эллиптических уравнений и систем второго порядка, имеющих решения с особенностями, порядок которых соответствует концевым точкам интервала. Эти примеры доказывают, что интервал правильного поведения порядка особенности установлен точно (в случае второго порядка).

\section{§1. Поведение решений в окрестности особой точки}

В области $\Omega \subset \mathbb{R}^{n}, n \geqslant 2$, рассматривается эллиптическая система

$$
\mathscr{L}^{i} u \equiv \operatorname{div}^{t_{i}} A^{i}\left(x, \mathscr{D}^{s} u\right)=0, \quad i=1, \ldots, N
$$

где $\mathscr{D}^{s} u=\left\{D^{k} u^{i}: k=0,1, \ldots, s_{i}, i=1, \ldots, N\right\}, s_{i}+t_{i}=2 m_{i}, s_{i}$ и $t_{i}$ - целые неотрицательные числа, $m_{i}$ - целые положительные числа; $D=\left(D_{1}, \ldots, D_{n}\right)$, $D_{j}=\partial / \partial x_{j}$. Функции $A^{i}$ измеримы и удовлетворяют следующему структурному условию: функции $B^{i}$, определяемые равенствами

$$
\Delta^{m_{i}} u^{i}+\operatorname{div}^{t_{i}} B^{i}\left(x, \mathscr{D}^{s} u\right)=\varkappa^{i} \operatorname{div}^{t_{i}} A^{i}\left(x, \mathscr{D}^{s} u\right)
$$

при некоторых нормирующих множителях $\varkappa^{i}>0$, удовлетворяют оценке

$$
\sum_{i=1}^{N}\left|B^{i}(x, \xi)\right|^{2} \leqslant \sum_{i=1}^{N} K_{i}^{2}\left|\xi_{s}^{i}\right|^{2}, \quad K_{i}<1
$$

Здесь $B^{i}$ имеет размерность $n^{t_{i}}, D^{s_{i}} u^{i}$ и, соответственно, $\xi_{s}^{i}$ - размерность $n^{s_{i}}$, так что

$$
\left|B^{i}\right|^{2}=\sum_{j_{1}=1}^{n} \cdots \sum_{j_{t_{i}}=1}^{n}\left(B_{j_{1} \ldots j_{i}}^{i}\right)^{2}, \quad\left|\xi_{s}^{i}\right|^{2}=\sum_{j_{1}=1}^{n} \cdots \sum_{j_{s_{i}}=1}^{n}\left(\xi_{j_{1} \ldots j_{s_{i}}}^{i}\right)^{2} .
$$

Такая запись мультииндексов, игнорируюшая совпадение смешанных производных при перемене порядка дифференцирования, выбрана ради простоты формы структурного условия (2). Ниже как решения, так и пробные функции в интегральном тождестве таковы, что смешанные производные при перемене порядка дифференцирования совпадают, поэтому $B^{i}$ можно считать симметрическими по всем внешним индексам $j_{l}$, а также симметрическими по всем индексам $j_{l}$ аргумента $\xi_{s}^{i}$.

Условие (2) введено нами в работе [5]. Оно обеспечивает коэрцитивность оператора $\varkappa \mathscr{L} u$ в паре с оператором $\Delta^{\left(s_{i}-t_{i}\right) / 2} u^{i}$ в пространстве $H^{s}=\prod_{i=1}^{N} W_{2}^{s_{i}}[6]$. Условие (2) обобщает условие Кордеса для недивергентных уравнений с одной стороны и стандартные структурные условия для дивергентных эллиптических систем с другой. 
Действительно, пусть $s=2 m, t=0, N=1$. Рассмотрим недивергентный оператор

$$
\sum_{|\alpha|=2 m} A_{\alpha}(x) D^{\alpha} u
$$

$A_{\alpha} \in L_{\infty}$, мультиндексы понимаются в смысле (3), т.е. $\alpha=\left(j_{1}, \ldots, j_{|\alpha|}\right), j_{l} \in$ $\{1, \ldots, n\}$. В этом случае $K$ (наименьшее возможное $K$ при различных $\varkappa$ ) явно выражается через коэффициенты:

$$
\begin{aligned}
K & =\sup _{x} \min _{\varkappa} \max _{|\xi|=1} \sum_{|\alpha|,|\beta|=m}\left(\delta_{\alpha \beta}-\varkappa A_{\alpha \beta}(x)\right) \xi_{\alpha \beta} \\
& =\sup _{x} \min _{\varkappa}\left(\sum_{|\alpha|,|\beta|=m}\left(\delta_{\alpha \beta}-\varkappa A_{\alpha \beta}\right)^{2}\right)^{1 / 2} \\
& =\sup _{x} \min _{\varkappa}\left(n^{m}-2 \varkappa \sum_{|\alpha|=m} A_{\alpha \alpha}+\varkappa^{2} \sum_{|\alpha|,|\beta|=m} A_{\alpha \beta}^{2}\right)^{1 / 2} \\
& =\sup _{x}\left(n^{m}-\left(\sum_{|\alpha|=m} A_{\alpha \alpha}\right)^{2}\left(\sum_{|\alpha|,|\beta|=m} A_{\alpha \beta}^{2}\right)^{-1}\right)^{1 / 2}
\end{aligned}
$$

где $\varkappa$ - нормирующий множитель, $\delta_{\alpha \beta}$ - символ Кронекера. Поэтому условие $K<1$ совпадает с классическим условием Кордеса для матрицы коэффициентов $\left(A_{\alpha}\right)$, рассматриваемой как квадратная матрица $n^{m} \times n^{m}$. Напомним, что условие Кордеса $K_{\varepsilon}[3]$ для матрицы $\left(a_{i j}\right), i, j=1, \ldots, M$, имеет вид

$$
\sum_{i=1}^{M} a_{i i} \geqslant\left((M-1+\varepsilon) \sum_{i, j=1}^{M} a_{i j}^{2}\right)^{1 / 2}, \quad \varepsilon>0 .
$$

Для дивергентных систем $\left(s_{i}=t_{i}=m_{i}, N \geqslant 1\right)$ условие (2) соответствует стандартной паре структурных условий

$$
\left(A(x, \xi), \xi_{m}\right) \geqslant c_{1}\left|\xi_{m}\right|^{2}, \quad|A(x, \xi)| \leqslant c_{2}\left|\xi_{m}\right|,
$$

причем $\max _{i} K_{i}^{2} \leqslant 1-c_{1}^{2} / c_{2}^{2}$. Действительно, при $s_{i}=t_{i}$ имеем $B^{i}(x, \xi)=$ $\varkappa^{i} A^{i}(x, \xi)-\xi_{m}^{i}$ и из (2) находим

$$
\left(1-K^{2}\right)\left|\xi_{m}\right|^{2}+|\varkappa A(x, \xi)|^{2} \leqslant 2\left(\varkappa A(x, \xi), \xi_{m}\right),
$$

$K=\max _{i} K_{i}<1$, откуда следуют условия (4) для $\varkappa A$. В обратную сторону, если вьполнено (4), то

$$
\begin{aligned}
|B(x, \xi)|^{2} & =\left|\varkappa A(x, \xi)-\xi_{m}\right|^{2}=\left|\xi_{m}\right|^{2}-2 \varkappa\left(A, \xi_{m}\right)+\varkappa^{2}|A|^{2} \\
& \leqslant\left(1-2 \varkappa c_{1}+\varkappa^{2} c_{2}^{2}\right)\left|\xi_{m}\right|^{2}
\end{aligned}
$$


откуда при $\varkappa=c_{1} / c_{2}^{2}$ следует (2) с $K^{2}=1-c_{1}^{2} / c_{2}^{2}$. Отметим, что точное выражение $K$ для дивергентных систем через собственные числа матрицы $\partial A(x, \xi) / \partial \xi_{m}$, рассматриваемой как квадратная матрица $n^{m} N \times n^{m} N$, получено в [7; гл. $\left.1, \S 1.3\right]$.

Ниже устанавливается, что решения системы (1) ведут себя "правильно" на некотором интервале шкалы весовых пространств $H^{s}\left(\Omega ;|x|^{a}\right)$. Для описания зависимости этого интервала от показателей $K_{i}$ рассмотрим сингулярные интегральные операторы $T^{s, t}=D^{s} \Delta^{-(s+t) / 2} \operatorname{div}^{t}$, где $s+t$ - четное число, $\Delta^{-1}$ - оператор с символом $-|\zeta|^{-2}, D=\left(D_{1}, \ldots, D_{n}\right), D_{j}=\partial / \partial x_{j}$, div $=D^{\prime}$. Эти операторы переводят функции размерности $n^{t}$ в функции размерности $n^{s}$. Обозначим через $T_{a}^{s, t}$ норму $T^{s, t}$ в весовом пространстве $L_{2}\left(\mathbb{R}^{n} ;|x|^{a}\right)$ (строго говоря, из одной декартовой степени этого пространства в другую). Поскольку $T^{s, t}$ являются композициями проекторов Рисса, $T_{a}^{s, t}<\infty$ при $a \in(-n, n)$ и $T_{a}^{s, t} \rightarrow \infty$ при $|a| \rightarrow n-0$. По равенству Парсеваля в $L_{2}$ без веса $T_{0}^{s, t}=1$. Также $T_{a}^{s, t}$ логарифмиически выпукло по $a$ по интерполяционной теореме Рисса-Торина [8; гл. 5]. Поскольку $K_{i}<1$, эти свойства $T_{a}^{s, t}$ обеспечивают, что уравнение

$$
T_{a}^{s_{i}, t_{i}}=\frac{1}{K_{i}}
$$

имеет один корень $a_{i}^{*} \in(0, n)$ и один корень $a_{* i} \in(-n, 0)$ (если $K_{i}=0$, полагаем $a_{i}^{*}=n, a_{* i}=-n ;$ случай, когда $K_{i}=0$ при всех $i=1, \ldots, N$ и система совпадает с полилапласианом, не рассматривается).

Обозначим $a^{*}=\min _{i} a_{i}^{*}, a_{*}=\max _{i} a_{* i}$. Легко видеть, что

$$
T_{a}^{s_{i}, t_{i}}<\frac{1}{K_{i}}, \quad a \in\left(a_{*}, a^{*}\right)
$$

(следует из логарифмической выпуклости $T_{a}^{s, t}$ с учетом $T_{0}^{s, t}=1<1 / K_{i}$ ). Отметим, что $a^{*} \rightarrow n, a_{*} \rightarrow-n$ при $\max _{i} K_{i} \rightarrow 0$.

Обозначим $U_{R}=\left\|D^{s} u ; L_{2}\left(Q_{R}\right)\right\|^{2}, Q_{R}=\{x: R<|x|<2 R\},\left|D^{s} u\right|^{2}=$ $\sum_{i=1}^{N}\left|D^{s_{i}} u^{i}\right|^{2}$, и введем множество $F=\{n-2 t+2 j: j=0,1, \ldots, t-1\}, t=$ $\max _{i} t_{i}$. Буквой $c$ будем обозначать различные несущественные положительные константы.

TЕорема 1. Пусть $и \in H_{\mathrm{loc}}^{s}(\Omega \backslash 0)$ - решение системь (1) с изолированной особенностью в точке $0 \in \Omega$. Тогда при $R \rightarrow 0$ справедливо одно из следующих утверждений:

1) $R^{a} U_{R} \rightarrow \infty$ при любом $a<a^{*}$;

2) для некоторого $j \in\{0,1, \ldots, t-1\}$ такого, что $n-2 t+2 j \in\left(a_{*}, a^{*}\right)$,

$$
c_{1} \leqslant R^{n-2 t+2 j} U_{R} \leqslant c_{2},
$$

константы могут быть выцбраны так, что $c_{2} / c_{1} \leqslant c\left(n, s_{i}, t_{i}, K_{i}\right)$;

3) $R^{a} U_{R} \rightarrow 0$ при любом $a>a_{*}$.

Доказательство этой и следующих теорем будет получено в $\S 2$.

Отметим, что если $\Phi$ - фундаментальное решение полилапласиана $\Delta^{(s+t) / 2}$, то функция $D^{j} \Phi$ удовлетворяет утверждению 2 ). 
Грубо говоря, теорема утверждает, что если порядок особенности решения попадает в некоторый интервал, определяемый показателями $K_{i}$ из $(2)$, то порядок особенности может быть только такой же, как у одного из сингулярных решений полилапласиана. Интервал может как расширяться до $(-n, n)$ при $K_{i} \rightarrow 0$, так и сужаться до произвольно малой окрестности нуля при $K_{i} \rightarrow 1$. Для сравнения укажем, что для линейных эллиптических систем с постоянньми коэффициентами такой результат справедлив на интервале $(-\infty, \infty)$ (если ограничиваться степенной шкалой).

В случаях 1) и 3) теоремы 1 могут быть получены более точные оценки решения. А именно справедлива

Теорема 2. В случае 1) теоремы 1 если $a^{*} \notin F$, то справедливо одно из утверждений:

$\left.1^{*}\right) c_{1} \leqslant R^{a^{*}} U_{R} \leqslant c_{2}$,

$\left.1^{* *}\right) \liminf \operatorname{in}_{R \rightarrow 0} R^{a^{*}} U_{R}=\infty$,

а если $a^{*} \in F$, то выполнено

$\left.1_{F}\right) R^{a^{*}} U_{R} \geqslant c(\ln 1 / R)^{-2}$.

В случае 3) теоремы 1 если $a_{*} \notin F$, то справедливо одно из утверэсдений:

$\left.3^{*}\right) c_{1} \leqslant R^{a_{*}} U_{R} \leqslant c_{2}$,

$\left.3^{* *}\right) \lim \sup _{R \rightarrow 0} R^{a_{*}} U_{R}=0$,

аесли $a_{*} \in F$, то выполнено

$\left.3_{F}\right) R^{a_{*}} U_{R} \leqslant c(\ln 1 / R)^{2}$.

В случаях $\left.1^{*}\right)$ и $\left.3^{*}\right)$ константы можсно вылбрать так, что $c_{2} / c_{1} \leqslant c\left(n, s_{i}, t_{i}, K_{i}\right)$.

Из теорем 1, 2 вытекают следующие условия устранимости особой точки. Устранимость понимается как справедливость интегрального тождества с пробными функциями из $H_{\text {comp }}^{t}(\Omega)$, не финитными в нуле.

СЛЕДСТВИЕ 1. Пусть $u \in H_{\mathrm{loc}}^{s}(\Omega \backslash 0)$ - решение системы (1) с особенностью в точке $0 \in \Omega$. Пусть $a_{*}<n-2 t$, а также

$$
\begin{aligned}
\liminf _{R \rightarrow 0} R^{\min \left\{n-2 t, a^{*}\right\}} U_{R}=0, & a^{*} \neq n-2 t \\
\liminf _{R \rightarrow 0} R^{n-2 t}\left(\ln \frac{1}{R}\right)^{2} U_{R}=0, & a^{*}=n-2 t .
\end{aligned}
$$

Тогда особенность устранима.

Действительно, из условий следствия на $U_{R}$ по теореме 1 при $a^{*}>n-2 t$ или по теоремам 2,1 при $a^{*} \leqslant n-2 t$ следует, что $R^{a} U_{R} \rightarrow 0$ при $R \rightarrow 0$ для любого $a>a_{*}$. Поскольку $a_{*}<0$, это дает $u \in H_{\mathrm{loc}}^{s}(\Omega)$.

Проверим справедливость интегрального тождества. Пусть пробная функция $v \in C_{0}^{\infty}(\Omega)$. Введем срезаюшую функцию $\varphi \in C_{0}^{\infty}\left(B_{R}\right): 0 \leqslant \varphi \leqslant 1, \varphi=1$ в $B_{R / 2}$, 
$\left|D^{j} \varphi\right| \leqslant c_{j} R^{-j}, B_{R}=\{x:|x|<R\}$. Представляя единицу в виде $1=(1-\varphi)+\varphi$, имеем $v(1-\varphi) \in C_{0}^{\infty}(\Omega \backslash 0)$. В интегральном тождестве получаем

$$
\begin{aligned}
& \left|\int A\left(x, \mathscr{D}^{s} u\right) D^{t} v d x\right|=\left|\int A\left(x, \mathscr{D}^{s} u\right) D^{t}(v \varphi) d x\right| \\
& \quad \leqslant c\left\|D^{s} u ; L_{2}\left(B_{R}\right)\right\| \sum_{j=0}^{t} R^{-j}\left\|D^{t-j} v ; L_{2}\left(B_{R}\right)\right\| \leqslant c R^{-t+n / 2-a / 2}
\end{aligned}
$$

с произвольньм $a>a_{*}$. При выборе $a \in\left(a_{*}, n-2 t\right)$ правая часть стремится к нулю при $R \rightarrow 0$, что доказывает справедливость интегрального тождества с пробными функциями из $C_{0}^{\infty}(\Omega)$.

Для пробных функций из $H_{\text {comp }}^{t}(\Omega)$ интегральное тождество следует теперь из плотности $C_{0}^{\infty}$ в этом пространстве с учетом $u \in H_{\mathrm{loc}}^{s}(\Omega)$.

Рассмотрим поведение решений на бесконечности.

Теорема 3. Пусть $u \in H_{\mathrm{loc}}^{s}(\Omega)$ - решение системь (1) в области $\Omega$ с ограниченным дополнением. Тогда при $R \rightarrow \infty$ справедливо одно из следующих утверждений:

1) $R^{a} U_{R} \rightarrow 0$ при любом $a<a^{*}$

2) для некоторого $j \in\{0,1, \ldots, t-1\}$ такого, что $n-2 t+2 j \in\left(a_{*}, a^{*}\right)$,

$$
c_{1} \leqslant R^{n-2 t+2 j} U_{R} \leqslant c_{2}
$$

константы могут быть выбраны так, что $c_{2} / c_{1} \leqslant c\left(n, s_{i}, t_{i}, K_{i}\right)$;

3) $R^{a} U_{R} \rightarrow \infty$ при любом $a>a^{*}$.

В случаях 1) и 3), как и выше, имеют место более точные оценки решения. А именно справедлива

Теорема 4. В случае 1) теоремъ 3 если а* $\notin F$, то справедливо одно из утверждений:

$\left.1^{*}\right) c_{1} \leqslant R^{a^{*}} U_{R} \leqslant c_{2}$

$\left.1^{* *}\right) \lim \sup _{R \rightarrow \infty} R^{a^{*}} U_{R}=0$,

а если $a^{*} \in F$, то выполнено

$\left.1_{F}\right) R^{a^{*}} U_{R} \leqslant c(\ln R)^{2}$.

В случае 3$)$ теоремь 3 если $a_{*} \notin F$, то справедливо одно из утверждений:

$\left.3^{*}\right) c_{1} \leqslant R^{a_{*}} U_{R} \leqslant c_{2}$,

$\left.3^{* *}\right) \liminf \operatorname{in}_{R \rightarrow \infty} R^{a_{*}} U_{R}=\infty$,

а если $a_{*} \in F$, то выполнено

$\left.3_{F}\right) R^{a_{*}} U_{R} \geqslant c(\ln R)^{-2}$.

В случаях $\left.1^{*}\right)$ и $\left.3^{*}\right)$ константы можно выбрать так, что $c_{2} / c_{1} \leqslant c\left(n, s_{i}, t_{i}, K_{i}\right)$.

Из оценок $D^{s} u$, даваемых теоремами $1-4$, достаточно стандартно следуют соответствующие оценки для самого решения. Так, например, если имеет место п. 2) 
теоремы 1 , то при $R \rightarrow 0$

$$
\begin{array}{ll}
R^{n+2 j-2 t-2 s_{i}} \int_{Q_{R}}\left|u^{i}\right|^{2} d x \leqslant c, & s_{i}<n+j-t, \\
R^{n+2 j-2 t-2 s_{i}} \int_{Q_{R}}\left|u^{i}-p^{i}\right|^{2} d x \leqslant c\left(\ln \frac{1}{R}\right)^{2}, & s_{i} \geqslant n+j-t,
\end{array}
$$

полином $p^{i}$ степени $\operatorname{deg} p^{i}<s_{i}+t-j-n$ определяется условиями

$$
\left.D^{\gamma}\left(u^{i}-p^{i}\right)\right|_{x=0}=0, \quad|\gamma|<s_{i}+t-j-n
$$

Действительно, обозначая $a_{j}=n-2 t+2 j$ и выбирая $b=a_{j}-1$, из оценки п. 2$)$ теоремы 1 находим

$$
R^{a_{j}-b} \int_{Q_{R \rho}}\left|D^{s} u\right|^{2} r^{b} d x \leqslant c c_{2},
$$

$r=|x|, Q=Q_{R \rho}=\{x: R<r<2 \rho\}, 0<R<\rho<\frac{1}{2} \operatorname{dist}(0, \partial \Omega)$. По неравенству Харди (17)

$$
\begin{aligned}
R^{a_{j}-b} & \int_{Q_{R \rho}}\left|D^{s_{i}-1} u^{i}\right|^{2} r^{b-2} d x \\
& \leqslant c R^{a_{j}-b}\left(\int_{Q_{R \rho}}\left|D^{s_{i}} u^{i}\right|^{2} r^{b} d x+\int_{Q_{\rho}}\left|D^{s_{i}-1} u^{i}\right|^{2} r^{b-2} d x\right) \\
& \leqslant c c_{2}+c R^{a_{j}-b} \rho^{b-2}\left\|D^{s_{i}-1} u^{i}\right\|_{Q_{\rho}}^{2}
\end{aligned}
$$

где $\|f\|_{Q}=\left\|f ; L_{2}(Q)\right\|$. Если $s_{i}<n+j-t$, применяя неравенство Харди $s_{i}$ раз, получаем

$$
\begin{aligned}
R^{a_{j}-2 s_{i}} \int_{Q_{R}}\left|u^{i}\right|^{2} d x & \leqslant c R^{a_{j}-b} \int_{Q_{R \rho}}\left|u^{i}\right|^{2} r^{b-2 s_{i}} d x \\
& \leqslant c c_{2}+c R^{a_{j}-b} \sum_{b=1}^{s_{i}} \rho^{b-2 l}\left\|D^{s_{i}-l} u^{i}\right\|_{Q_{\rho}}^{2}
\end{aligned}
$$

откуда следует искомая оценка при $R \rightarrow 0$ с учетом $a_{j}>b$.

Если $s_{i} \geqslant n+j-t$, обозначая $k=n+j-t$, аналогично находим для $l=k-1$

$$
R^{a_{j}-2 l} \int_{Q_{R}}\left|D^{s_{i}-l} u^{i}\right|^{2} d x \leqslant c
$$

при этом $a_{j}-2 l=2-n$. Для оценки производных порядка $s_{i}-k$ используем следующий аналог неравенства Харди (17) для критического показателя:

$$
\left|\|f\|_{-n, Q_{R}}-\|f\|_{-n, Q_{\rho}}\right| \leqslant\left(\ln \frac{\rho}{R}\right)^{1 / 2}\|D f\|_{2-n, Q_{R} \rho}
$$

где $\|f\|_{a, Q}=\left\|f ; L_{2}\left(Q ; r^{a}\right)\right\|$. 
Из имеющейся оценки производных порядка $s_{i}-k+1$ по этому неравенству немедленно следует

$$
\begin{aligned}
& R^{-n} \int_{Q_{R}}\left|D^{s_{i}-k} u^{i}\right|^{2} d x \\
& \quad \leqslant c \ln \frac{\rho}{R} \int_{Q_{R \rho}}\left|D^{s_{i}-k+1} u^{i}\right|^{2} r^{2-n} d x+c \rho^{-n} \int_{Q_{\rho}}\left|D^{s_{i}-k} u^{i}\right|^{2} d x \\
& \quad \leqslant c\left(\ln \frac{\rho}{R}\right)^{2}+c \rho^{-n} \int_{Q_{\rho}}\left|D^{s_{i}-k} u^{i}\right|^{2} d x
\end{aligned}
$$

что дает искомую оценку при $s_{i}=n+j-t$.

Если $s_{i}>n+j-t$, полученная оценка $D^{s_{i}-k} u^{i}$ обеспечивает существование в нуле производных $D^{\gamma} u^{i}(0),|\gamma|<s_{i}-k$, что однозначно определяет полином $p^{i}$. Выберем $b=a_{j}+1$ и применим неравенство Харди (17) с $R=0$ к производным функции $u_{0}^{i}=u^{i}-p^{i}$. Находим

$$
\begin{aligned}
& \rho^{a_{j}-2 k-2} \int_{Q_{\rho}}\left|D^{s_{i}-k-1} u_{0}^{i}\right|^{2} d x \leqslant c \rho^{a_{j}-b} \int_{Q_{\rho}}\left|D^{s_{i}-k-1} u_{0}^{i}\right|^{2} r^{b-2 k-2} d x \\
& \leqslant c \rho^{a_{j}-b} \int_{B_{2 \rho}}\left|D^{s_{i}-k} u^{i}\right|^{2} r^{b-2 k} d x \leqslant c \rho^{a_{j}-b} \int_{0}^{\rho} r^{b-a_{j}}\left(\ln \frac{1}{r}\right)^{2} \frac{d r}{r} \leqslant c\left(\ln \frac{1}{\rho}\right)^{2} .
\end{aligned}
$$

Итерация этой оценки $s_{i}-k$ раз завершает доказательство.

При выполнении каких-либо добавочных условий, обеспечивающих гёльдеровость обобщенного решения системы (1), из теорем следуют соответствующие поточечные оценки для $u$. Так, например, если вьполнено условие $s_{i}>\left(n+a_{*}\right) / 2$, обеспечивающее гёльдеровость $u^{i}$ внутри области определения, см. [9], то в случае 2) теоремы 1 при $|x| \rightarrow 0$

$$
\begin{array}{ll}
\left|u^{i}(x)\right| \leqslant c|x|^{s_{i}+t-n-j}, & s_{i}<n+j-t, \\
\left|u^{i}(x)-p^{i}(x)\right| \leqslant c|x|^{s_{i}+t-n-j} \ln \frac{1}{|x|}, & s_{i} \geqslant n+j-t .
\end{array}
$$

В частности, в дивергентном случае при совпадении порядков системы по всем $i$, т.е. в случае $s_{i}=t_{i}=m, i=1, \ldots, N$, имеем $a_{*}=-a^{*}$ ввиду самосопряженности оператора $T^{m, m}$, и из условия $n-2 m+2 j \in\left(a_{*}, a^{*}\right)$ следует $m-\left(n+a_{*}\right) / 2>j$, что обеспечивает гёльдеровость $D^{j} u$, см. [9]. Поэтому для систем с $s_{i}=t_{i}=m$ если имеет место случай 2 ) теоремы 1 , то при $|x| \rightarrow 0$

$$
\begin{array}{ll}
\left|D^{j} u(x)\right| \leqslant c|x|^{2 m-n}, & 2 m-j<n, \\
\left|D^{j}(u(x)-p(x))\right| \leqslant c|x|^{2 m-n} \ln \frac{1}{|x|}, & 2 m-j \geqslant n .
\end{array}
$$

Укажем явные оценки для чисел $a^{*}, a_{*}$, которые точны при $s+t=2$. На интервале $b \in(-n, n)$ определим функцию

$$
M_{s, t}(b)= \begin{cases}M_{1}(b)^{t} M_{2}(b)^{(s-t) / 2}, & s \geqslant t, \\ M_{1}(b)^{s} M_{2}(-b)^{(t-s) / 2}, & s \leqslant t\end{cases}
$$




$$
\begin{aligned}
M_{1}(b) & =\sup _{j=1,2, \ldots} M_{1}\left(b, \lambda_{j}\right), \quad M_{2}(b)=\sup _{j=0,1, \ldots} M_{2}\left(b, \lambda_{j}\right), \quad \lambda_{j}=j(j+n-2), \\
M_{1}(b, \lambda) & =1+b^{2}\left(\lambda^{1 / 2}+\lambda^{-1 / 2} \frac{(n-2)^{2}-b^{2}}{4}\right)^{-2}, \\
M_{2}(b, \lambda) & =1+b\left(\lambda(b+n-3)+(n-1)\left(\frac{b+n-4}{4}\right)^{2}\right)\left(\lambda+\frac{n-b}{2} \frac{n+b-4}{2}\right)^{-2} .
\end{aligned}
$$

Для каждого конкретного $b$ супремум легко вычисляется при исследовании функции на максимум по $\lambda$ с помощью дифференцирования. Для функции $M_{1}$ интервал $(0, n)$ разбивается на конечное количество интервалов числами $b_{j}=\left((n-2)^{2}-\right.$ $\left.4\left(\lambda_{j} \lambda_{j+1}\right)^{1 / 2}\right)^{1 / 2}, j$ - натуральные числа такие, что $\lambda_{j} \lambda_{j+1}<((n-2) / 2)^{4}$. Обозначим через $J_{n}$ количество этих интервалов, в частности $J_{n}=1$ при $n \leqslant 8$. Тогда, полагая $b_{0}=n, b_{J_{n}}=0$, имеем

$$
M_{1}(b)=M_{1}\left(b, \lambda_{j}\right), \quad b_{j} \leqslant|b|<b_{j-1},
$$

в частности

$$
\begin{gathered}
M_{1}(b)=M_{1}\left(b, \lambda_{1}\right)=1+b^{2}(n-1)\left(\frac{4}{n^{2}-b^{2}}\right)^{2}, \\
\left((n-2)^{2}-4(2 n(n-1))^{1 / 2}\right)^{1 / 2} \leqslant|b|<n, \quad n>8 ; \quad|b|<n, \quad n \leqslant 8 .
\end{gathered}
$$

Для функции $M_{2}$

$$
M_{2}(b)= \begin{cases}1+b(n-1)\left(\frac{2}{n-b}\right)^{2}, & 0 \leqslant b<n, \\ 1, & 3-n \leqslant b \leqslant 0, \\ M_{2}\left(b, \lambda_{2}\right), & -n<b \leqslant \min \{0 ; 1+\sqrt{3}-n\},\end{cases}
$$

а также при $n \geqslant 3$ сушествует возрастающая последовательность $b_{j} \rightarrow 3-n$, $j \geqslant 2, b_{j}>1+\sqrt{3}-n$, такая, что $M_{2}(b)=M_{2}\left(b, \lambda_{j}\right), b_{j-1}<b \leqslant b_{j}$, $b_{1}=-n$. В отличие от функции $M_{1}$ здесь числа $b_{j}$, являющиеся корнями уравнений $M_{2}\left(b, \lambda_{j}\right)=M_{2}\left(b, \lambda_{j+1}\right)$, не выражаются в радикалах.

Обозначим через $b_{i}^{*} \in(0, n), b_{* i} \in(-n, 0)$ корни уравнения $M_{s_{i}, t_{i}}(b)=1 / K_{i}^{2}$ (если $K_{i}=0$, полагаем $b_{i}^{*}=n, b_{* i}=-n$ ). Положим $b^{*}=\min _{i} b_{i}^{*}, b_{*}=\max _{i} b_{* i}$.

В [6] установлено, что $T_{a}^{t, s} \leqslant\left(M_{s, t}(a)\right)^{1 / 2}$ и при $s+t=2$ имеет место равенство. Отсюда сразу следует, что $a^{*} \geqslant b^{*}, a_{*} \leqslant b_{*}$, т.е. $\left(b_{*}, b^{*}\right) \subset\left(a_{*}, a^{*}\right)$.

В некоторых случаях уравнение $M_{s, t}(b)=1 / K^{2}$ тривиально сводится к биквадратному или квадратному и решается в радикалах:

1) $s=t=m:$

$$
b^{*}=-b_{*}=\min _{j=1,2, \ldots}\left\{\left(\frac{4 \lambda_{j}}{1-K^{2 / m}}+(n-2)^{2}\right)^{1 / 2}-\left(\frac{4 \lambda_{j} K^{2 / m}}{1-K^{2 / m}}\right)^{1 / 2}\right\} ;
$$

2 ) та же формула для $b^{*}$ с заменой $m$ на $s$ при $s<t$ четном, если $b^{*} \in[0, n-3]$;

и для $b_{*}$ с заменой $m$ на $t$ при $s>t$ четном, если $b_{*} \in[3-n, 0]$;

3) $t=0, m=s / 2$ :

$$
b^{*}=\frac{1}{1-K^{2 / m}}\left(\left(n-K^{2 / m}\right)^{1 / 2}-K^{1 / m}(n-1)^{1 / 2}\right)^{2} ;
$$

та же формула для $-b_{*}$ при $s=0, m=t / 2$. 
ЗАмЕчАНИЕ 1 . Для проверки условия $a \in\left(b_{*}, b^{*}\right)$ нет необходимости находить $b^{*}, b_{*}$ явно: в силу вьпуклости $M_{s, t}$ оно равносильно

$$
K_{i}^{2} M_{s_{i}, t_{i}}(a)<1, \quad i=1, \ldots, N .
$$

\section{§2. Оценки решений с бистепенным весом}

Доказательства теорем основаны на интегральных оценках решения с бистепенным весом типа $\min \left\{(r / \tau)^{a} ;(r / \tau)^{b}\right\}, r=|x|, \tau>0$ - параметр. Обозначим $Q=Q_{R \rho}=\{x: R<|x|<2 \rho\}, 2 R \leqslant \rho, Q_{R}=\{x: R<|x|<2 R\} ;$ $\|u\|_{\omega, G}=\left\|\omega^{1 / 2} u ; L_{2}(G)\right\|$, где $\omega(x)$ - произвольный неотрицательный вес, $G$ - область, при $\omega=1$ или $G=\mathbb{R}^{n}$ соответствуюший индекс опускаем, в случае степенного веса $\omega=r^{a}$ будем обозначать $\|f\|_{a}=\left\|r^{a / 2} f\right\|$. Введем срезаюшую функцию $\varphi \in C_{0}^{\infty}(Q): 0 \leqslant \varphi \leqslant 1, \varphi=1$ при $2 R<|x|<\rho,\left|D^{j} \varphi(x)\right| \leqslant c_{j}|x|^{-j}$. Будем предполагать $\tau \in(R, \rho)$.

Рассмотрим сначала случай $t=\max _{i} t_{i}>0$.

Лемма 1. Пусть $u \in H^{s}(Q)$ - решение системь (1) в $Q$. Тогда для произвольных чисел $a, b$, удовлетворяющих условию $a_{*} \leqslant b \leqslant a \leqslant a^{*}$, и произвольного $\tau \in(R, \rho)$ найдутся функция $v=\left(v^{1}, \ldots, v^{N}\right)$ и функиия $w$ некоторой размерности такие, что

$$
\begin{aligned}
& \mu(a)\left\|\left(\frac{r}{\tau}\right)^{-a / 2} \omega \varphi D^{s} u\right\|^{2}+\mu(b)\left\|\left(\frac{r}{\tau}\right)^{-b / 2} \omega \varphi D^{s} u\right\|^{2} \\
& \quad \leqslant c\left(\left\|D^{s} u\right\|\left(\sum_{i=1}^{N}\left(\left\|D^{t_{i}} v^{i}\right\|+\left\|r^{-t_{i}}\left(v^{i}-P^{i}\right)\right\|\right)+\left\|r^{-1}\left(w-p_{w}\right)\right\|\right)\right)_{Q_{R} \cup Q_{\rho}},(7)
\end{aligned}
$$

$\mu(a)=\min \left\{1-a / a^{*}, 1-a / a_{*}\right\}, \omega(r)=\left((r / \tau)^{-a}+(r / \tau)^{-b}\right)^{-1}, P-$ произвольньий полином степени $\operatorname{deg} P^{i}<t_{i}, p_{w}-$ произвольный полином нулевой степени той же размерности, что $w$. При этом функции $v, w$ при произвольном $q \in(-n, n)$ удовлетворяют неравенству

$$
\left\|D^{t} v\right\|_{q}+\|D w\|_{q} \leqslant c\left\|\omega \varphi D^{s} u\right\|_{q}
$$

əде $\|f\|_{q}=\left\|r^{q / 2} f\right\|$, константа ограничена, если $n-|q|$ отделено от нуля.

ЗАмечАнИЕ 2 . При $b>a_{*}, a<a^{*}$ левая часть (7) оценивает сверху норму $\left\|\varphi D^{s} u\right\|_{\omega}^{2}$, поскольку $(r / \tau)^{-a} \omega(r) \asymp 1$ при $r<\tau,(r / \tau)^{-b} \omega(r) \asymp 1$ при $r>\tau$.

ДокАЗАТЕЛЬСТво. Не ограничивая общности, будем считать $K_{i}>0$ при всех $i=1, \ldots, N$. Действительно, поскольку $a_{i}^{*} \rightarrow n, a_{* i} \rightarrow-n$ при $K_{i} \rightarrow 0$, замена в (2) нулевого $K_{i}$ достаточно малым положительным $K_{i}$ не меняет значений $a^{*}, a_{*}$.

Положим

$$
v^{i}=K_{i}^{2} \Delta^{-m_{i}} \operatorname{div}^{s_{i}}\left(\omega \varphi D^{s_{i}} u^{i}\right),
$$

где $\Delta^{-m}$ - оператор с символом $\left(-|\zeta|^{2}\right)^{-m}$. Оценка (8) для $v$ следует из ограниченности проекторов Рисса, композицией которых является оператор $D^{t_{i}} \Delta^{-m_{i}} \operatorname{div}^{s_{i}}$, в весовом пространстве $L_{2}\left(\mathbb{R}^{n} ;|x|^{q}\right), q \in(-n, n)[10]$. Подставим в интегральное 
тождество для системы $(1)$ пробную функцию $v_{0}=(v-p) \varphi$. При $q=0$ имеем $D^{t} v \in L_{2}$, так что подстановка допустима. По условию (2) и неравенству Коши из интегрального тождества следует

$$
\begin{aligned}
\sum_{i}\left|\left(\Delta^{m_{i}} u^{i}, v_{0}^{i}\right)\right| & \leqslant \int\left(\sum_{i}\left|B^{i}\right|^{2}\right)^{1 / 2}\left(\sum_{i}\left|D^{t_{i}} v_{0}^{i}\right|^{2}\right)^{1 / 2} d x \\
& \leqslant\left(\sum_{i} K_{i}^{2}\left\|D^{s_{i}} u^{i}\right\|_{\omega}^{2}\right)^{1 / 2}\left(\sum_{i}\left\|D^{t_{i}} v^{i}\right\|_{1 / \omega}^{2}\right)^{1 / 2}+c U V
\end{aligned}
$$

где мы обозначили

$$
U V=\left(\left\|D^{s} u\right\| \sum_{i=1}^{N}\left(\left\|D^{t_{i}} v^{i}\right\|+\left\|r^{-t_{i}}\left(v^{i}-P^{i}\right)\right\|\right)\right)_{Q_{R} \cup Q_{\rho}},
$$

модули производных понимаются аналогично (3).

Преобразуем левую часть. Пусть при рассматриваемом $i$ для определенности $s_{i}, t_{i}$ четные положительные. Опуская для краткости векторный индекс, имеем

$$
\left|\left(\Delta^{m} u, v_{0}\right)\right|=\int \Delta^{s / 2} u \Delta^{t / 2} v_{0} d x \geqslant \int \varphi \Delta^{s / 2} u \Delta^{t / 2} v d x-c U V .
$$

Перебросим с помошью интегрирования по частям $\Delta^{s / 2}$ на $v, \operatorname{a~div}^{s}$, содержашееся в функции $v$, на $u$, но так, чтобы возможно меньше дифференцировать $\varphi$. По определению $v$ имеем $\Delta^{t / 2} v=K^{2} \operatorname{div}^{s} \Delta^{-s / 2}\left(\omega \varphi D^{s} u\right)$. Введем обозначение: $v_{1}=K^{2} \operatorname{div}^{s-1} \Delta^{-s / 2}\left(\omega \varphi D^{s} u\right)$ - вектор-функция размерности $n$. Дважды интегрируя по частям, получаем

$$
\begin{aligned}
& \int \varphi \Delta^{s / 2} u \Delta^{t / 2} v d x=-\int\left(\varphi D \Delta^{s / 2} u+D \varphi \Delta^{s / 2} u\right)\left(v_{1}-p_{1}\right) d x \\
& =\int\left(\varphi D^{2} \Delta^{s / 2-1} u D v_{1}+\left(D^{2} \Delta^{s / 2-1} u-\Delta^{s / 2} u\right)\left(v_{1}-p_{1}\right) D \varphi\right) d x \\
& \geqslant \int \varphi D \Delta^{s / 2-1} D u D v_{1} d x-c\left(\left\|D^{s} u\right\|\left\|r^{-1}\left(v_{1}-p_{1}\right)\right\|\right)_{Q_{R} \cup Q_{\rho}}
\end{aligned}
$$

где $p_{1}-$ произвольньй полином нулевой степени размерности $n$. Для функции $v_{1}$ ввиду ограниченности проекторов Рисса в $L_{2}\left(\mathbb{R}^{n},|x|^{q}\right),|q|<n$, имеем

$$
\left\|D v_{1}\right\|_{q} \leqslant c\left\|\omega \varphi D^{s} u\right\|_{q} .
$$

Опять дважды проинтегрировав по частям, находим

$$
\int \varphi \Delta^{s / 2-1} D u D v_{1} d x \geqslant \int \varphi \Delta^{s / 2-1} D^{2} u v_{2} d x-c\left(\left\|D^{s} u\right\|\left\|r^{-1}\left(w_{2}-p_{2}\right)\right\|\right)_{Q_{R}} \cup Q_{\rho},
$$

где $v_{2}=K^{2} \operatorname{div}^{s-2} \Delta^{1-s / 2}\left(\omega \varphi D^{s} u\right)$ - вектор-функция размерности $n^{2}$,

$$
w_{2}=D \operatorname{div}^{s-2} \Delta^{-s / 2}\left(\omega \varphi D^{s} u\right)
$$


- вектор-функция размерности $n^{3}, p_{2}$ - произвольный полином нулевой степени размерности $n^{3}$. За $s$ шагов получим

$$
\int \varphi \Delta^{s / 2} u \Delta^{s / 2} v d x \geqslant K^{2}\left\|\varphi D^{s} u\right\|_{\omega}^{2}-c U W
$$

где мы обозначили

$$
U W=\left(\left\|D^{s} u\right\|\left\|r^{-1}\left(w-p_{w}\right)\right\|\right)_{Q_{R} \cup Q_{\rho}}
$$

и где для функции $w$ выполнено (8). Случай нечетных $s_{i}, t_{i}$ или четных $s_{i}, t_{i}$, когда одно из них равно нулю, рассматривается аналогично.

Оценим правую часть (9). Так как $1 / \omega$ - сумма двух степенных весов, находим (векторньй индекс опушен для краткости)

$$
\begin{aligned}
\left\|D^{t} v\right\|_{1 / \omega}^{2} & =K^{2}\left\|T^{t, s}\left(\omega \varphi D^{s} u\right)\right\|_{1 / \omega}^{2} \\
& \leqslant K^{2} \int\left(\left(T_{-a}^{t, s}\right)^{2}\left(\frac{r}{\tau}\right)^{-a}+\left(T_{-b}^{t, s}\right)^{2}\left(\frac{r}{\tau}\right)^{-b}\right)\left|\omega \varphi D^{s} u\right|^{2} d x .
\end{aligned}
$$

Отметим, что

$$
T_{-a}^{t, s}=T_{a}^{s, t}
$$

Действительно, пусть $f, g$ - вектор-функции размерностей $n^{t}$ и $n^{s}$ соответственно, $\|f\|_{a}=\|g\|_{-a}=1$, где $\|f\|_{a}=\left\|f ; L_{2}\left(\mathbb{R}^{n} ;|x|^{a}\right)\right\|$. Тогда

$$
\begin{aligned}
T_{-a}^{t, s} & =\sup _{g}\left\|T^{t, s} g\right\|_{-a}=\sup _{f, g} \int f T^{t, s} g d x=\sup _{f, g} \int g T^{s, t} f d x \\
& =\sup _{f}\left\|T^{s, t} f\right\|_{a}=T_{a}^{s, t},
\end{aligned}
$$

где центральное равенство следует из $\left(T^{t, s}\right)^{*}=T^{s, t}$. Учитывая, что $(r / \tau)^{-a}+$ $(r / \tau)^{-b}=1 / \omega$, находим

$$
\begin{aligned}
\left\|D^{t} v\right\|_{1 / \omega}^{2} \leqslant & K^{2}\left\|\varphi D^{s} u\right\|_{\omega}^{2}-K^{2}\left(1-\left(K T_{a}^{s, t}\right)^{2}\right)\left\|\left(\frac{r}{\tau}\right)^{-a / 2} \omega \varphi D^{s} u\right\|^{2} \\
& -K^{2}\left(1-\left(K T_{b}^{s, t}\right)^{2}\right)\left\|\left(\frac{r}{\tau}\right)^{-b / 2} \omega \varphi D^{s} u\right\|^{2}
\end{aligned}
$$

Поскольку $T_{a}^{s, t}$ как функция от $а$ логарифмически выпукла и $T_{0}^{s, t}=1$, имеем при $a \in\left(0, a^{*}\right)$

$$
K_{i} T_{a}^{s_{i}, t_{i}} \leqslant\left(K_{i} T_{0}^{s_{i}, t_{i}}\right)^{1-a / a^{*}}\left(K_{i} T_{a^{*}}^{s_{i}, t_{i}}\right)^{a / a^{*}} \leqslant K_{i}^{1-a / a^{*}}
$$

и далее

$$
1-K_{i} T_{a}^{s_{i}, t_{i}} \geqslant\left(1-\frac{a}{a^{*}}\right)\left(1-K_{i}\right) .
$$

Аналогичная оценка справедлива при $a \in\left(a_{*}, 0\right)$. Из (13) получаем

$$
\left\|D^{t} v\right\|_{1 / \omega}^{2} \leqslant K^{2}\left\|\varphi D^{s} u\right\|_{\omega}^{2}-K^{2}(1-K) U_{\mu},
$$


где мы обозначили

$$
U_{\mu}=\mu(b)\left\|\left(\frac{r}{\tau}\right)^{-b / 2} \omega \varphi D^{s} u\right\|^{2}+\mu(a)\left\|\left(\frac{r}{\tau}\right)^{-a / 2} \omega \varphi D^{s} u\right\|^{2} .
$$

Применяя в (9) эту оценку и оценку (12), находим

$$
\sum_{i} K_{i}^{2} U_{\omega}^{i}-\left(\sum_{i} K_{i}^{2} U_{\omega}^{i}\right)^{1 / 2}\left(\sum_{i} K_{i}^{2}\left(U_{\omega}^{i}-\left(1-K_{i}\right) U_{\mu}^{i}\right)\right)^{1 / 2} \leqslant c U V+c U W
$$

г де $U_{\omega}^{i}=\left\|\varphi D^{s_{i}} u^{i}\right\|_{\omega}^{2}$. По элементарному неравенству $\alpha-(\alpha(\alpha-\beta))^{1 / 2} \geqslant \beta / 2$, $0 \leqslant \beta \leqslant \alpha$, отсюда следует (7).

Оценим правую часть (7) при соответствующем выборе полинома $P$. Обозначим $F=\{n-2 t+2 j: j=0,1, \ldots, t-1\}, t=\max _{i} t_{i}$, и занумеруем интервалы, на которые множество $F$ разбивает вешественную прямую: $I_{0}=(-\infty, n-2 t)$, $I_{j}=(n-2 t+2 j-2, n-2 t+2 j), j=1, \ldots, t-1, I_{t}=(n-2, \infty)$.

Лемма 2. Если а $\notin F$, то для функиии $v^{i} \in H^{t_{i}}(Q)$ существует полином $P^{i}$ степени $\operatorname{deg} P^{i}<t_{i}$ такой, что одновременно выполнено

$$
\begin{gathered}
\left\|r^{-t_{i}}\left(v^{i}-P^{i}\right)\right\|_{-a, Q_{R}} \leqslant \frac{c}{d_{+}(a, F)^{1 / 2}}\left\|D^{t_{i}} v^{i}\right\|_{-a, Q}, \\
\left\|r^{-t_{i}}\left(v^{i}-P^{i}\right)\right\|_{-a, Q_{\rho}} \leqslant \frac{c}{d_{-}(a, F)^{1 / 2}}\left\|D^{t_{i}} v^{i}\right\|_{-a, Q},
\end{gathered}
$$

$d_{+}(a, F)=\min \{b-a: b \in F, b>a\}$ (полагаем $d_{+}=1$ при $\left.a>F\right), d_{-}(a, F)=$ $\min \{a-b: b \in F, b<a\}$ (полагаем $d_{-}=1$ при $\left.a<F\right)$. Зависимость полинома от а исчерпывается зависимостью от $j$, определяемого условием $а \in I_{j}$ (т.е. для различных а из одного $I_{j}$ полином один и тот жее).

ДокАЗАТЕЛЬство. Используем неравенство Харди в виде

$$
\begin{gathered}
\frac{|n+b|}{2}\|f\|_{b, Q}^{2}+\|f\|_{b, G_{1}}^{2} \leqslant \frac{2}{|n+b|}\|D f\|_{b+2, Q}^{2}+2\|f\|_{b, G_{2}}^{2}, \\
G_{1}=Q_{R}, \quad G_{2}=Q_{\rho} \text { при } \quad b>-n ; \quad G_{1}=Q_{\rho}, \quad G_{2}=Q_{R} \quad \text { при } \quad b<-n .
\end{gathered}
$$

Оно следует из равенства $0=\int_{R}^{2 \rho}\left(f^{2} r^{b+n} \phi\right)^{\prime} d r$ при оценке слагаемого с $2 f^{\prime} f$ по неравенству Юнга, $\phi(r)$ - срезающая функция: $\phi=1$ при $2 R \leqslant r \leqslant \rho, \phi=r / R-1$ при $R \leqslant r \leqslant 2 R, \phi=2-r / \rho$ при $\rho \leqslant r \leqslant 2 \rho$.

Определим полином $P^{i}$ условиями

$$
\int_{Q_{R}} D^{\gamma}\left(v^{i}-P^{i}\right) d x=0, \quad|\gamma|<j_{i} ; \quad \int_{Q_{\rho}} D^{\gamma}\left(v^{i}-P^{i}\right) d x=0, \quad j_{i} \leqslant|\gamma|<t_{i},
$$

$j_{i}=t_{i}-t+j, j$ определяется условием $a \in I_{j}$.

Оценим норму по $Q_{R}$. Для функции $z=v^{i}-P^{i}$ по неравенству Пуанкаре

$$
\left\|r^{-t} z\right\|_{-a, Q_{R}} \leqslant c\left\|r^{1-t} D z\right\|_{-a, Q_{R}} \leqslant \cdots \leqslant c\left\|r^{j-t} D^{j} z\right\|_{-a, Q_{R}}
$$


(индекс $i$ опускаем для краткости) и далее по неравенству Харди (17)

$$
\begin{aligned}
d_{+}(a, F)^{1 / 2}\left\|r^{j-t} D^{j} z\right\|_{-a, Q_{R}} & \leqslant 2\left\|r^{j-t+1} D^{j+1} z\right\|_{-a, Q}+2\left\|r^{j-t} D^{j} z\right\|_{-a, Q_{\rho}} \\
& \leqslant c\left\|r^{j-t+1} D^{j+1} z\right\|_{-a, Q} \leqslant \cdots \leqslant\left\|D^{t} z\right\|_{-a, Q}
\end{aligned}
$$

что доказывает (15).

Оценим норму по $Q_{\rho}$. Не ограничивая обшности, полагаем $d_{-}(a, F) \leqslant 1$, так как оценка для $d_{-}>1$ следует из оценки для $d_{-}=1$. Действительно, если $d_{-}(a, F)>1$, выбирая $b<a$ такое, что $d_{-}(b, F)=1$ и $a, b$ принадлежат одной компоненте $\mathbb{R} \backslash F$, из оценки для $d_{-}=1$ находим

$$
\begin{aligned}
\left\|r^{-t} z\right\|_{-a, Q_{\rho}} & \leqslant \rho^{(b-a) / 2}\left\|r^{-t} z\right\|_{-b, Q_{\rho}} \leqslant c \rho^{(b-a) / 2}\left\|D^{t} z\right\|_{-b, Q} \\
& =c\left\|\left(\frac{r}{\rho}\right)^{(a-b) / 2} D^{t} v\right\|_{-a, Q} \leqslant c\left\|D^{t} v\right\|_{-a, Q} .
\end{aligned}
$$

Пусть $d_{-}(a, F) \leqslant 1$. Положим $b=n-2 t+2 j-3$, где $j$ из условия $a \in I_{j}$. Понятно, что $b \in I_{j-1}, b<a$. По неравенству Харди (17) и неравенству Пуанкаре в $Q_{R}$

$$
\begin{aligned}
\left\|r^{-t} z\right\|_{-b, Q_{\rho}} & \leqslant c\left\|r^{1-t} D z\right\|_{-b, Q}+c\left\|r^{-t} z\right\|_{-b, Q_{R}} \leqslant c\left\|r^{1-t} D z\right\|_{-b, Q} \\
& \leqslant \cdots \leqslant c\left\|r^{j-t-1} D^{j-1} z\right\|_{-b, Q} \\
& \leqslant c\left\|r^{j-t} D^{j} z\right\|_{-b, Q}+c\left\|r^{j-t-1} D^{j-1} z\right\|_{-b, Q_{\rho}} .
\end{aligned}
$$

Для первого слагаемого по неравенству Харди и неравенству Пуанкаре находим

$$
\left\|r^{j-t} D^{j} z\right\|_{-b, Q} \leqslant \frac{c}{d_{+}(b, F)}\left\|D^{t} z\right\|_{-b, Q} .
$$

Учитывая, что $d_{+}(b, F)=1$ и $b<a$, получаем

$$
\begin{aligned}
\left\|r^{j-t} D^{j} z\right\|_{-b, Q} & \leqslant c\left\|D^{t} z\right\|_{-b, Q} \\
& \leqslant c \rho^{(a-b) / 2}\left\|\left(\frac{r}{\rho}\right)^{(a-b) / 2} D^{t} z\right\|_{-a, Q} \leqslant c \rho^{(a-b) / 2}\left\|D^{t} z\right\|_{-a, Q} .
\end{aligned}
$$

Для последнего слагаемого в (20)

$$
\left\|r^{j-t-1} D^{j-1} z\right\|_{-b, Q} \leqslant c \rho^{(a-b) / 2}\left\|r^{j-t-1} D^{j-1} z\right\|_{-a, Q_{\rho}}
$$

и далее по неравенству Харди и неравенству Пуанкаре

$$
\begin{aligned}
d_{-}(a, F)^{1 / 2}\left\|r^{j-t-1} D^{j-1} z\right\|_{-a, Q_{\rho}} & \leqslant 2\left\|r^{j-t} D^{j} z\right\|_{-a, Q}+2\left\|r^{j-t-1} D^{j-1} z\right\|_{-a, Q_{R}} \\
& \leqslant c\left\|r^{j-t} D^{j} z\right\|_{-a, Q} \leqslant c\left\|D^{t} z\right\|_{-a, Q}
\end{aligned}
$$

последнее неравенство устанавливается аналогично (19) с учетом $d_{+}(a, F) \geqslant 1$. Поскольку в левой части (20)

$$
\left\|r^{-t} z\right\|_{-b, Q_{\rho}} \geqslant \rho^{(a-b) / 2}\left\|r^{-t} z\right\|_{-a, Q_{\rho}}
$$

это завершает доказательство (16). 
Лемма 3. Пусть для некоторой функиии $и \in H^{s}(Q)$ и некоторого интервала $\left(a_{*}, a^{*}\right) \Subset(-n, n)$ справедливо утверждение леммы 1 . Если числа $a, b$, $a_{*}<b \leqslant a<a^{*}$, принадлежат одной компоненте связности множества $\mathbb{R} \backslash F, m o$

$$
\begin{gathered}
\mu(a)\left\|\left(\frac{r}{\tau}\right)^{-a / 2} \omega \varphi D^{s} u\right\|^{2}+\mu(b)\left\|\left(\frac{r}{\tau}\right)^{-b / 2} \omega \varphi D^{s} u\right\|^{2} \\
\leqslant \frac{c}{d_{+}(a, F) \mu(a)} \omega(R) U_{R}+\frac{c}{d_{-}(b, F) \mu(b)} \omega(\rho) U_{\rho},
\end{gathered}
$$

əде $U_{R}=\left\|D^{s} u ; L_{2}\left(Q_{R}\right)\right\|^{2}, \omega(r)=\left((r / \tau)^{-a}+(r / \tau)^{-b}\right)^{-1}, \tau \in(R, \rho)$.

ЗАмЕчАниЕ 3. При фиксированных $a, b$ (если нас не интересует зависимость констант от расстояния от $a, b$ до $\left.a_{*}, a^{*}, F\right)$ по замечанию 2

$$
\left\|D^{s} u\right\|_{\omega, Q}^{2} \leqslant c \omega(R) U_{R}+c \omega(\rho) U_{\rho} .
$$

ДокаЗАтельСтво. Оценим члены с $v-P$ в правой части (7). Полином $P$ определим условиями (18), $j$ из условия $a, b \in I_{j}$. Применяя лемму 2, в кольце $Q_{R}$ находим

$$
\begin{aligned}
\left\|D^{s} u\right\|_{Q_{R}}\left\|r^{-t}(v-P)\right\|_{Q_{R}} & \leqslant c\left(\frac{R}{\tau}\right)^{a / 2}\left\|D^{s} u\right\|_{Q_{R}}\left\|\left(\frac{r}{\tau}\right)^{-a / 2} r^{-t}(v-P)\right\|_{Q_{R}} \\
& \leqslant \frac{c}{d_{+}(a, F)^{1 / 2}} \omega(R)^{1 / 2} U_{R}^{1 / 2}\left\|\left(\frac{r}{\tau}\right)^{-a / 2} D^{t} v\right\|_{Q},
\end{aligned}
$$

где мы учли $\omega(r) \asymp(r / \tau)^{a}$ при $r<\tau$. Используя (8), получаем

$$
\text { idem } \leqslant \frac{c}{d_{+}(a, F)^{1 / 2}} \omega(R)^{1 / 2} U_{R}^{1 / 2}\left\|\left(\frac{r}{\tau}\right)^{-a / 2} \omega \varphi D^{s} u\right\| .
$$

В кольце $Q_{\rho}$ так же находим

$$
\left\|D^{s} u\right\|_{Q_{\rho}}\left\|r^{-t}(v-P)\right\|_{Q_{\rho}} \leqslant \frac{c}{d_{-}(b, F)^{1 / 2}} \omega(\rho)^{1 / 2} U_{\rho}^{1 / 2}\left\|\left(\frac{r}{\tau}\right)^{-b / 2} \omega \varphi D^{s} u\right\|,
$$

поскольку $\omega(r) \asymp(r / \tau)^{b}$ при $r>\tau$. Аналогичные оценки справедливы для членов в правой части $(7)$, содержаших $w$ (соответствуюшее множество $F_{w}$ содержит ровно одну точку $n-2)$, и для членов с $D^{t} v$. Теперь утверждение леммы следует из (7) при применении неравенства Юнга.

ЛЕмма 4. Пусть для некоторой функции $и \in H^{s}(Q)$ и некоторого интервала $\left(a_{*}, a^{*}\right) \subset(-n, n)$ справедливо утверждение леммы 1. Если числа $a, b$, $a_{*}<b<a<a^{*}$, такие, что $(b, a) \cap F=\{n-2 t+2 j\}, a, b \notin F$, то

$$
\begin{aligned}
& \left\|D^{s} u\right\|_{\omega, Q}^{2} \leqslant c\left(\frac{R}{\tau}\right)^{n-2 t+2 j} U_{R}+c \omega(\rho) U_{\rho}, \\
& \left\|D^{s} u\right\|_{\omega, Q}^{2} \leqslant c \omega(R) U_{R}+c\left(\frac{\rho}{\tau}\right)^{n-2 t+2 j} U_{\rho} .
\end{aligned}
$$


ДокАЗАТЕЛЬСТво. Члены в (7), содержащие $D^{t} v$, легко оцениваются по формуле (8). В (7) определим полином $P$ условиями (18). Поскольку $b \in I_{j}$, по лемме 2 и по формуле (8) находим

$$
\begin{aligned}
\left\|D^{s} u\right\|_{Q_{\rho}}\left\|r^{-t}(v-P)\right\|_{Q_{\rho}} & \leqslant c \omega(\rho)^{1 / 2} U_{\rho}^{1 / 2}\left\|\left(\frac{r}{\tau}\right)^{-b / 2} D^{t} v\right\| \\
& \leqslant \omega \omega(\rho)^{1 / 2} U_{\rho}^{1 / 2}\left\|\left(\frac{r}{\tau}\right)^{-b / 2} \omega \varphi D^{s} u\right\| .
\end{aligned}
$$

В кольце $Q_{R}$ по неравенству Пуанкаре

$$
\left\|r^{-t}(v-P)\right\|_{Q_{R}} \leqslant c\left\|r^{j-t} D^{j}(v-P)\right\|_{Q_{R}} .
$$

Для дальнейших оценок представим функцию $g=D^{j}(v-p)$ в виде

$$
g(r, \theta)=g\left(\frac{r \rho}{R}, \theta\right)-\int_{r}^{r \rho / R} \frac{\partial g}{\partial h}(h, \theta) d h
$$

$(r, \theta)$ - полярные координаты. Находим

$$
\left\|r^{j-t} g\right\|_{Q_{R}}^{2} \leqslant c\left(\frac{R}{\rho}\right)^{n-2 t+2 j}\left\|r^{j-t} g\right\|_{Q_{\rho}}^{2}+c R^{n-2 t+2 j} \int\left(\int_{R}^{2 \rho}|D g| d r\right)^{2} d \theta
$$

и далее

$$
\int\left(\int_{R}^{2 \rho}|D g| d r\right)^{2} d \theta \leqslant \iint_{R}^{2 \rho}|D g|^{2} r^{2 j-2 t+1+n} \frac{1}{\omega} d r d \theta \int_{R}^{2 \rho} r^{2 t-2 j-n-1} \omega d r .
$$

Поскольку $b<n-2 t+2 j<a, \tau \in(R, \rho)$, последний интеграл не превосходит $c \tau^{2 t-2 j-n}$, так что

$$
\left\|r^{j-t} g\right\|_{Q_{R}}^{2} \leqslant c\left(\frac{R}{\tau}\right)^{n-2 t+2 j}\left\|r^{j-t+1} D g\right\|_{1 / \omega, Q}^{2} .
$$

Поскольку $1 / \omega$ - сумма двух степенных весов, применяя неравенство Харди (17) и неравенство Пуанкаре, с учетом $a, b<n-2 t+2 j+2$ получаем

$$
\left\|r^{j-t+1} D^{j+1}(v-P)\right\|_{1 / \omega, Q} \leqslant c\left\|D^{t} v\right\|_{1 / \omega} \leqslant c\left\|\varphi D^{s} u\right\|_{\omega}
$$

последнее неравенство следует из (8). Таким образом,

$$
\left\|D^{s} u\right\|_{Q_{R}}\left\|r^{-t}(v-P)\right\|_{Q_{R}} \leqslant c\left(\frac{R}{\tau}\right)^{n / 2-t+j} U_{R}^{1 / 2}\left\|\varphi D^{s} u\right\|_{\omega} .
$$

Аналогичные оценки справедливы для членов в (7), содержаших $w$. Оценивая правую часть (7) по формулам (25), (26) и используя неравенство Юнга, получаем (23). 
Доказательство (24) аналогично, полином $P$ определяется условиями

$$
\int_{Q_{R}} D^{\gamma}\left(v^{i}-P^{i}\right) d x=0, \quad|\gamma| \leqslant j_{i} ; \quad \int_{Q_{\rho}} D^{\gamma}\left(v^{i}-P^{i}\right) d x=0, \quad j_{i}<|\gamma|<t_{i},
$$

$j_{i}=t_{i}-t+j$. Члены, связанные с $Q_{R}$, оцениваются по лемме 2 , так как $a \in I_{j+1}$. Для членов, связанных с $Q_{\rho}$, имеем

$$
\begin{aligned}
& \left\|\left(\frac{r}{\tau}\right)^{-b / 2} r^{-t}(v-P)\right\|_{Q_{\rho}} \leqslant c\left\|\left(\frac{r}{\tau}\right)^{-b / 2} r^{j-t} D^{j}(v-P)\right\|_{Q} \\
& \quad \leqslant c\left\|\left(\frac{r}{\tau}\right)^{-b / 2} r^{j-t+1} D^{j+1}(v-P)\right\|_{Q}+c\left\|\left(\frac{r}{\tau}\right)^{-b / 2} r^{j-t} D^{j}(v-P)\right\|_{Q_{\rho}} \\
& \quad \leqslant c\left\|\left(\frac{r}{\tau}\right)^{-b} D^{t} v\right\|+c \omega(\rho)^{-1 / 2}\left\|r^{j-t} D^{j}(v-P)\right\|_{Q_{\rho} .}
\end{aligned}
$$

Для оценки последнего члена, как и выше, используется представление функции $g=D^{j}(v-P)$ в виде

$$
g(r, \theta)=g\left(\frac{r R}{\rho}, \theta\right)+\int_{r R / \rho}^{r} \frac{\partial g}{\partial h}(h, \theta) d h .
$$

ДОКАЗАТЕЛЬСТВО ТЕОРЕМЫ 1 легко вьводится из лемм 1,4 при $\left(a_{*}, a^{*}\right) \cap$ $F \neq \varnothing$ и из лемм 1,3 в форме замечания 3 при $\left(a_{*}, a^{*}\right) \cap F=\varnothing$.

Рассмотрим сначала случай $\left(a_{*}, a^{*}\right) \cap F=\varnothing$. Если утверждение 1$)$ теоремы 1 не справедливо для решения $u$, то при некотором $b_{1}<a^{*}$ имеем

$$
\liminf _{R \rightarrow 0} R^{b_{1}} U_{R}<\infty
$$

Пусть $a_{*}<b<a<a^{*}$, причем $a \in\left(b_{1}, a^{*}\right)$. Пусть также $0<R<\tau<\rho<$ $\frac{1}{2} \operatorname{dist}(0, \partial \Omega)$. При $\omega(r)=\left((r / \tau)^{-a}+(r / \tau)^{-b}\right)^{-1}$ по лемме 1 и замечанию 3 имеем

$$
\left\|D^{s} u\right\|_{\omega, Q}^{2} \leqslant c \omega(R) U_{R}+c \omega(\rho) U_{\rho} \leqslant c\left(\frac{R}{\tau}\right)^{a} U_{R}+c\left(\frac{\rho}{\tau}\right)^{b} U_{\rho}
$$

При $R \rightarrow 0$ из (27) получаем

$$
\left\|D^{s} u\right\|_{\omega, B_{\rho}}^{2} \leqslant c\left(\frac{\rho}{\tau}\right)^{b} U_{\rho}
$$

$B_{\rho}=\{x: r<\rho\}$. Поскольку $\omega(r) \asymp 1$ в кольце $Q_{\tau}$, отсюда следует

$$
\tau^{b} U_{\tau} \leqslant c \rho^{b} U_{\rho},
$$

$0<\tau<\rho$. Ввиду произвольности $b>a_{*}$ это доказывает справедливость утверждения 3) теоремы 1 для $u$. 
Рассмотрим случай $\left(a_{*}, a^{*}\right) \cap F \neq \varnothing$. Если утверждение 1$)$ теоремы 1 не справедливо для решения $u$, то при некотором $b_{1}<a^{*}$ выполнено (27). Пусть $a_{j}=n-2 t+2 j$ - наибольший элемент множества $\left(a_{*}, a^{*}\right) \cap F$. Выберем $a, b$, $a_{*}<b<a<a^{*}$, так, что $(b, a) \cap F=\left\{a_{j}\right\}, a, b \notin F$ и $a>b_{1}$. Пусть $0<R<\tau<\rho<\frac{1}{2} \operatorname{dist}(0, \partial \Omega)$. По лемме 1 и оценке (24) леммы 4 имеем

$$
\left\|D^{s} u\right\|_{\omega, Q}^{2} \leqslant c\left(\frac{R}{\tau}\right)^{a} U_{R}+c\left(\frac{\rho}{\tau}\right)^{a_{j}} U_{\rho}
$$

При $R \rightarrow 0$ из (27) получаем

$$
\left\|D^{s} u\right\|_{\omega, B_{\rho}}^{2} \leqslant c\left(\frac{\rho}{\tau}\right)^{a_{j}} U_{\rho}
$$

и, поскольку $\omega(r) \asymp 1$ в кольце $Q_{\tau}$,

$$
\tau^{a_{j}} U_{\tau} \leqslant c \rho^{a_{j}} U_{\rho}
$$

Таким образом, выполнена оценка сверху утверждения 2) теоремы 1 с данным $j$. Если вьполнена также оценка снизу, при $\tau \rightarrow 0$ получаем

$$
c \rho^{a_{j}} U_{\rho} \geqslant \limsup _{\tau \rightarrow 0} \tau^{a_{j}} U_{\tau}>0 .
$$

Отсюда при $\rho \rightarrow 0$ следует

$$
c \liminf _{R \rightarrow 0} R^{a_{j}} U_{R} \geqslant \limsup _{R \rightarrow 0} R^{a_{j}} U_{R}
$$

что доказывает возможность выбора констант $c_{1}, c_{2}$ таким образом, что $c_{2} / c_{1} \leqslant$ $c(n, s, t, K)$.

Пусть оценка снизу утверждения 2) теоремы 1 с данным $j$ не выполнена для $u$, т.e.

$$
\liminf _{R \rightarrow 0} R^{a_{j}} U_{R}=0
$$

Выберем $a, b, a_{*}<b<a<a^{*}$, так, что $(b, a) \cap F=\left\{a_{j}\right\}, a, b \notin F$. По лемме 1 и оценке (23) леммы 4 имеем

$$
\left\|D^{s} u\right\|_{\omega, Q}^{2} \leqslant c\left(\frac{R}{\tau}\right)^{a_{j}} U_{R}+c\left(\frac{\rho}{\tau}\right)^{b} U_{\rho}
$$

При $R \rightarrow 0$ из (29) получаем

$$
\left\|D^{s} u\right\|_{\omega, B_{\rho}}^{2} \leqslant c\left(\frac{\rho}{\tau}\right)^{b} U_{\rho}
$$

и, поскольку $\omega(r) \asymp 1$ в кольце $Q_{\tau}$,

$$
\tau^{b} U_{\tau} \leqslant c \rho^{b} U_{\rho}
$$

Тем самым,

$$
\limsup _{R \rightarrow 0} R^{b} U_{R}<\infty
$$

и мы попали в ситуацию, аналогичную исходной, с той разницей, что интервал $\left(a_{*}, b\right)$ содержит на одну точку из $F$ менњше, чем интервал $\left(a_{*}, a^{*}\right)$. Ввиду конечности множества $F$ за конечное число шагов устанавливается отсутствие для решения других возможностей, кроме указанных в теореме 1.

Для уточнения оценок в утверждениях 1) и 3 ) теоремы 1 установим еше две технические леммы. 
Лемма 5. Пусть для некоторой функции $u \in H^{s}(Q)$ и некоторого интервала $\left(a_{*}, a^{*}\right) \Subset(-n, n)$ справедливо утверждение леммы 1. Если числа $a, b$ принадлежат одной компоненте связности множества $\mathbb{R} \backslash F u$ $a_{*}<b<a=a^{*}$ или $a_{*}=b<a<a^{*}$, mо

$$
\mu(a)\left\|D^{s} u\right\|_{\omega, Q_{R \tau}}^{2}+\mu(b)\left\|D^{s} u\right\|_{\omega, Q_{\tau \rho}}^{2} \leqslant c \omega(R) U_{R}+c \omega(\rho) U_{\rho} .
$$

ДокАЗАТЕЛЬСТво. Пусть $a_{*}<b<a=a^{*}$. Определим полином $P$ в (7) условиями (18), $j$ из условия $a, b \in I_{j}$. Для членов, связанных с $Q_{\rho}$, по лемме 2 и неравенству (8) находим

$$
\begin{gathered}
\left\|D^{s} u\right\|_{Q_{\rho}}\left\|r^{-t}(v-P)\right\|_{Q_{\rho}} \leqslant c \omega(\rho)^{1 / 2} U_{\rho}^{1 / 2}\left\|\left(\frac{r}{\tau}\right)^{-b / 2} r^{-t}(v-P)\right\|_{Q_{\rho}} \\
\leqslant c \omega(\rho)^{1 / 2} U_{\rho}^{1 / 2}\left\|\left(\frac{r}{\tau}\right)^{-b / 2} D^{t} v\right\| \leqslant c \omega(\rho)^{1 / 2} U_{\rho}^{1 / 2}\left\|\left(\frac{r}{\tau}\right)^{-b / 2} \omega \varphi D^{s} u\right\| .
\end{gathered}
$$

Для членов с $w$ и $D^{t} v$ оценка аналогична. Поскольку $\mu(b)>0$, применяя неравенство Юнга, получаем из (7)

$$
\left\|\left(\frac{r}{\tau}\right)^{-b / 2} \omega \varphi D^{s} u\right\|^{2} \leqslant c \omega(\rho) U_{\rho}+c U_{R}^{1 / 2}\left(\left\|D^{t} v\right\|+\left\|r^{-t}(v-P)\right\|+\left\|r^{-t}\left(w-p_{w}\right)\right\|\right)_{Q_{R}} .
$$

Для оценки членов, связанных с $Q_{R}$, выберем $\alpha>a$ такое, что $\alpha \in I_{j}, \alpha<n$, $2 a-\alpha \geqslant b$, и применим лемму 2 и оценку (8):

$$
\begin{aligned}
\left\|r^{-t}(v-P)\right\|_{Q_{R}} & \leqslant c\left(\frac{R}{\tau}\right)^{\alpha / 2}\left\|\left(\frac{r}{\tau}\right)^{-\alpha / 2} D^{t} v\right\|_{Q} \\
& \leqslant c\left(\frac{R}{\tau}\right)^{\alpha / 2}\left\|\left(\frac{r}{\tau}\right)^{-\alpha / 2} \omega \varphi D^{s} u\right\| \leqslant c\left(\frac{R}{\tau}\right)^{\alpha / 2}\left\|\varphi D^{s} u\right\|_{\sigma}
\end{aligned}
$$

$\sigma(r)=\left((r / \tau)^{\alpha-2 a}+(r / \tau)^{-b}\right)^{-1}$. Так как $2 a-\alpha, b$ принадлежат одной компоненте связности множества $\left(a_{*}, a^{*}\right) \backslash F$, вес $\sigma$ удовлетворяет условиям замечания 3 . Используя (22), получаем

$$
\left(\frac{R}{\tau}\right)^{\alpha}\left\|\varphi D^{s} u\right\|_{\sigma}^{2} \leqslant c\left(\frac{R}{\tau}\right)^{2 a} U_{R}+c\left(\frac{R}{\tau}\right)^{\alpha}\left(\frac{\rho}{\tau}\right)^{b} U_{\rho} .
$$

Оценивая аналогично члены с $w$ и $D^{t} v$, из (31) находим

$$
\left\|\left(\frac{r}{\tau}\right)^{-b / 2} \omega \varphi D^{s} u\right\|^{2} \leqslant c \omega(R) U_{R}+c \omega(\rho) U_{\rho}
$$

Поскольку $\omega(r) \asymp(r / \tau)^{b}$ при $r>\tau$, отсюда следует (30).

В случае $a_{*}=b<a<a^{*}$ доказательство полностью аналогично. 
Лемма 6. Пусть для некоторой функиии $u \in H^{s}(Q)$ и некоторого интервала $\left(a_{*}, a^{*}\right) \Subset(-n, n)$ справедливо утверждение леммы 1. Если числа $a, b$ такие, что $a_{*}<b<a=a^{*}$, $a^{*} \in F, u$ принадлежит той компоненте связности $\mathbb{R} \backslash F$, правый конеи которой равен $a^{*}$, то

$$
\left\|D^{s} u\right\|_{\omega, Q_{\tau \rho}}^{2} \leqslant c\left(1+\ln \frac{\tau}{R}\right)^{2} \omega(R) U_{R}+c \omega(\rho) U_{\rho} .
$$

Eсли $a_{*}=b<a<a^{*}, a_{*} \in F$, и а принадлежит той компоненте связности $\mathbb{R} \backslash F$, левый конец которой равен $a_{*}$, то

$$
\left\|D^{s} u\right\|_{\omega, Q_{R \tau}}^{2} \leqslant c \omega(R) U_{R}+c\left(1+\ln \frac{\rho}{\tau}\right)^{2} \omega(\rho) U_{\rho} .
$$

ДокАЗАТЕЛЬСТво. Пусть $a_{*}<b<a=a^{*}$. Положим $\alpha=a^{*}-1 /(1+\ln (\tau / R))$. Тогда $\alpha, b$ принадлежат одной компоненте связности $\mathbb{R} \backslash F$. Применяя лемму 3 с весом $\sigma(r)=\left((r / \tau)^{-\alpha}+(r / \tau)^{-b}\right)^{-1}$, получаем

$$
\begin{aligned}
& \mu(\alpha)\left\|\left(\frac{r}{\tau}\right)^{-\alpha / 2} \sigma \varphi D^{s} u\right\|^{2}+\mu(b)\left\|\left(\frac{r}{\tau}\right)^{-b} \sigma \varphi D^{s} u\right\|^{2} \\
& \leqslant c\left(1+\ln \frac{\tau}{R}\right)^{2} \sigma(R) U_{R}+c \sigma(\rho) U_{\rho},
\end{aligned}
$$

поскольку $d_{+}(\alpha, F)=1 /(1+\ln (\tau / R)), \mu(\alpha)=1 /(1+\ln (\tau / R)) a^{*}$. Учитьвая, что

$$
\sigma(R)<\left(\frac{R}{\tau}\right)^{a}\left(\frac{R}{\tau}\right)^{-1 /(1+\ln (\tau / R))} \leqslant e\left(\frac{R}{\tau}\right)^{a}
$$

и $\sigma(r) \asymp(r / \tau)^{b}$ при $r>\tau$, получаем первую оценку леммы.

Случай $a_{*}=b<a<a^{*}$ рассматривается полностью аналогично.

ДОКАЗАТЕЛЬСТВО ТЕОРЕМЫ 2. Пусть для решения $u$ справедливо утверждение 1$)$ теоремы 1 . Пусть $a^{*} \notin F$. Предположим, что $\left.1^{* *}\right)$ не выполнено, т.е.

$$
\liminf _{R \rightarrow 0} R^{a^{*}} U_{R}<\infty .
$$

Выберем числа $a, b$ так, что $a_{*}<b<a=a^{*}$ и $a, b$ принадлежат одной компоненте связности множества $\mathbb{R} \backslash F$. По лемме 5 получаем

$$
\left\|D^{s} u\right\|_{\omega, Q_{\tau \rho}}^{2} \leqslant c\left(\frac{R}{\tau}\right)^{a} U_{R}+c\left(\frac{\rho}{\tau}\right)^{b} U_{\rho}
$$

откуда при $R \rightarrow 0$ следует

$$
\tau^{a}\left\|D^{s} u\right\|_{\omega, Q_{\tau \rho}}^{2} \leqslant c \liminf _{R \rightarrow 0} R^{a} U_{R}+c \tau^{a-b} \rho^{b} U_{\rho} .
$$


Если $\liminf$ положителен, то, учитывая, что $\omega \asymp 1$ в $Q_{\tau}$ и $a>b$, получаем при $\tau \rightarrow 0$

$$
\limsup _{R \rightarrow 0} R^{a} U_{R} \leqslant c \liminf _{R \rightarrow 0} R^{a} U_{R}
$$

что доказывает $\left.1^{*}\right)$ и возможность выбора констант так, что $c_{2} / c_{1} \leqslant c(n, s, t, K)$.

Предположение, что $1^{*}$ ) такжене выполнено, т.е. lim inf равен нулю, дает оценку

$$
\tau^{b} U_{\tau} \leqslant c \rho^{b} U_{\rho}
$$

$\tau \in(0, \rho), b<a^{*}$, которая противоречит предположению о справедливости утверждения 1) теоремы 1.

В случае $a^{*} \in F$ выберем $a, b$ так, что $a_{*}<b<a=a^{*}$ и $b$ принадлежит той компоненте $\mathbb{R} \backslash F$, правый конец которой равен $a^{*}$. По лемме 6

$$
\left\|D^{s} u\right\|_{\omega, Q_{\tau \rho}}^{2} \leqslant c\left(1+\ln \frac{\tau}{R}\right)^{2}\left(\frac{R}{\tau}\right)^{a} U_{R}+c\left(\frac{\rho}{\tau}\right)^{b} U_{\rho} .
$$

Предположение, что $1_{F}$ ) не вьполняется, т.е.

$$
\liminf _{R \rightarrow 0}\left(\ln \frac{1}{R}\right)^{2} R^{a} U_{R}=0,
$$

приводит к оценке

$$
\tau^{b} U_{\tau} \leqslant c \rho^{b} U_{\rho}
$$

$\tau \in(0, \rho)$. Поскольку $b<a^{*}$, это противоречит утверждению 1$)$ теоремы 1 , что доказывает справедливость $\left.1_{F}\right)$.

Случай, когда решение $u$ удовлетворяет утверждению 3$)$ теоремы 1 , рассматривается полностью аналогично.

Рассмотрим случай $t=0$, т.е. $t_{i}=0$ при всех $i=1, \ldots, N$.

При $t=0$ оценка леммы 1 создает недостаточньй базис для доказательства теорем в случае $a^{*} \geqslant n-2$. А именно наличие члена с $w$ в правой части не позволяет исключить случай 2) теоремы 1 с $R^{n-2}$. Этот пробел восполняет следующая лемма.

Лемма 7. Пусть $t=0$, и пусть $u \in H^{s}(Q)$ - решение системь (1) в $Q$. Тогда для произвольных чисел $a, b$, удовлетворяющих условию $a_{*} \leqslant b \leqslant a \leqslant a^{*}, u$ произвольного $\tau \in(R, \rho)$ найдется функиия $w$ некоторой размерности такая, чmo

$$
\begin{aligned}
& \mu(a)\left\|\left(\frac{r}{\tau}\right)^{-a / 2} \omega \varphi D^{s} u\right\|^{2}+\mu(b)\left\|\left(\frac{r}{\tau}\right)^{-b / 2} \omega \varphi D^{s} u\right\|^{2} \\
& \quad \leqslant c\left(\left\|D^{s} u\right\|\left\|r^{-1}\left(w-p_{w}\right)\right\|\right)_{Q_{R} \cup Q_{\rho}} \\
& \text { idem } \leqslant c\left(\left\|r^{-1} D^{s-1}\left(u-P_{u}\right)\right\|\|D w\|\right)_{Q_{R} \cup Q_{\rho}},
\end{aligned}
$$

$P_{u}-$ произвольный полином степени $\operatorname{deg} P_{u}^{i}<s_{i}, p_{w}-$ полином нулевой степени, $w$ удовлетворяет оченке (8).

При $n=2$ для $a, b$ таких, что $-\varepsilon<b \leqslant a<\varepsilon, \varepsilon=\varepsilon(K)>0$, выполнено

$$
\left\|D^{s} u\right\|_{\omega, Q}^{2} \leqslant c \omega(R) U_{R}+c \omega(\rho) U_{\rho} .
$$


ДокАЗАТЕЛЬСтво. Выбирая функцию $v$ так же, как при доказательстве леммы 1, получаем вместо (9)

$$
\sum_{i}\left|\left(\Delta^{m_{i}} u^{i}, v_{0}^{i}\right)\right| \leqslant\left(\sum_{i} K_{i}^{2}\left\|\varphi D^{s_{i}} u^{i}\right\|_{\omega}^{2}\right)^{1 / 2}\left(\sum_{i}\left\|v^{i}\right\|_{1 / \omega}^{2}\right)^{1 / 2} .
$$

Рассуждая дальше так же, как при доказательстве леммы 1 , приходим к оценке (32).

Для получения оценки (33) будем интегрировать по частям в левой части неравенства в другом порядке по сравнению с (10):

$$
\begin{aligned}
& \int \varphi \Delta^{m} u v d x=-\int D \Delta^{m-1}\left(u-P_{u}\right)(\varphi D v+D \varphi v) d x \\
& \quad=\int\left(\varphi D^{2} \Delta^{m-1} u D v_{1}+D \Delta^{m-1}\left(u-P_{u}\right)\left(D \varphi D v_{1}-D \varphi \operatorname{div} v_{1}\right)\right) d x \\
& \geqslant \int \varphi D^{2} \Delta^{m-1} u D v_{1} d x-c\left(\left\|r^{-1} D^{2 m-1}\left(u-P_{u}\right)\right\|\left\|D v_{1}\right\|\right)_{Q_{R} \cup Q_{\rho}},
\end{aligned}
$$

где $v_{1}=K^{2} \operatorname{div}^{s-1} \Delta^{-m}\left(\omega \varphi D^{s} u\right)$ (напомним, что $s=2 m$, поскольку $t=0$ ) и для $v_{1}$ вьполнено (11). Рассуждая дальше так же, как при доказательстве леммы 1 , приходим к оценке (33).

Докажем (34). При $t=0$ из (1) по условию (2) следует

$$
\left|\Delta^{m} u\right|^{2} \leqslant K^{2}\left|D^{2 m} u\right|^{2},
$$

$K=\max _{i} K_{i}<1,\left|\Delta^{m} u\right|^{2}=\sum_{i}\left(\Delta^{m_{i}} u_{i}\right)^{2}$, модуль производной понимается аналогично (3). Поэтому для неотрицательного финитного в $Q$ веса $\sigma=\omega \varphi$

$$
\left\|\Delta^{m} u\right\|_{\sigma}^{2}-K^{2}\left\|D^{2 m} u\right\|_{\sigma}^{2} \leqslant 0 .
$$

Интегрирование по частям дает для произвольной функции $v$

$$
\begin{aligned}
\left\|D^{2} v\right\|_{\sigma}^{2}-\|\Delta v\|_{\sigma}^{2} & =\int\left(\Delta v D_{i} v D_{i} \sigma-D_{i} D_{j} v D_{i} v D_{j} \sigma\right) d x \\
& =\int\left(|D v|^{2} \Delta \sigma-D_{i} v D_{j} v D_{i} D_{j} \sigma\right) d x
\end{aligned}
$$

по повторяющимся индексам идет суммирование. Перейдем в полярные координаты $(r, \theta)$ и разложим $v$ в ряд по гармоническим полиномам:

$$
v(r, \theta)=\sum_{j=0}^{\infty} v_{j}(r) r^{j} Y_{j}(\theta)
$$

где $\left\{Y_{j}\right\}$ - полная ортонормированная система собственных функций оператора Бельтрами на окружности (косинусы и синусы), $Y_{j}$ соответствует собственному числу $j^{2}$ (индекс, указывающий кратность $Y_{j}$, опускаем). Получаем

$$
\left\|D^{2} v\right\|_{\sigma}^{2}-\|\Delta v\|_{\sigma}^{2}=\sum_{j=0}^{\infty} \int\left(v_{j}^{\prime 2} \sigma^{\prime}+j(j-1) v_{j}^{2}\left(\frac{\sigma^{\prime}}{r}\right)^{\prime}\right) r^{2 j} d r
$$


штрих обозначает дифференцирование по $r$. Так как по условию леммы $-\varepsilon<b \leqslant$ $a<\varepsilon$, имеем

что дает

$$
\left|\omega^{\prime}\right| \leqslant \varepsilon \frac{\omega}{r}, \quad\left|\left(\frac{\omega^{\prime}}{r}\right)^{\prime}\right|<\varepsilon(2+\varepsilon) \frac{\omega}{r^{3}},
$$

$$
\begin{aligned}
\left\|D^{2} v\right\|_{\sigma}^{2}-\|\Delta v\|_{\sigma}^{2} \leqslant & \varepsilon \sum_{j=0}^{\infty} \int\left(v_{j}^{\prime 2}+(2+\varepsilon) j(j-1) v_{j}^{2} r^{-2}\right) r^{2 j-1} \omega \varphi d r \\
& +c \sum_{j=0}^{\infty}\left(\int_{R}^{2 R}+\int_{\rho}^{2 \rho}\right)\left(v_{j}^{\prime 2}+j(j-1) v_{j}^{2} r^{-2}\right) r^{2 j-1} \omega d r
\end{aligned}
$$

Записьвая вторую производную в полярных координатах, находим

$$
\begin{aligned}
\left|D^{2} v\right|^{2}= & \left(D_{k} D_{l} v\right)^{2}=v^{\prime \prime 2}+r^{-2} v^{\prime 2}+2 r^{-2} v_{\theta}^{\prime 2}+2 r^{-3} v^{\prime} v_{\theta \theta} \\
& -4 r^{-3} v_{\theta}^{\prime} v_{\theta}+r^{-4} v_{\theta}^{2}+r^{-4}\left(D_{\theta, k} D_{\theta, l} v\right)^{2},
\end{aligned}
$$

где $v_{\theta}=\partial v / \partial \theta, D_{\theta, k}=r D_{k}-r^{-1} x_{k} x_{i} D_{i}$, по повторяющимся индексам идет суммирование. На окружности имеем также

$$
\int\left(D_{\theta, k} D_{\theta, l} v(r, \theta)\right)^{2} d \theta=\int\left(v_{\theta \theta}^{2}+v_{\theta}^{2}\right) d \theta .
$$

При разложении $v$ в ряд по гармоническим полиномам получаем при каждом $r$

$$
\begin{aligned}
\int\left|D^{2} v(r, \theta)\right|^{2} d \theta= & \sum_{j=0}^{\infty}\left(\left(r^{j} v_{j}\right)^{\prime \prime 2}+\left(1+2 j^{2}\right) v_{j}^{\prime 2} r^{2 j-2}\right. \\
& \left.+2 j(j-1)(2 j-1) v_{j}^{\prime} v_{j} r^{2 j-3}+3 j^{2}(j-1)^{2} v_{j}^{2} r^{2 j-4}\right) .
\end{aligned}
$$

Сравнивая соответствующие коэффициенты здесь и в (36), находим

$$
(1-\varepsilon)\left\|D^{2} v\right\|_{\sigma}^{2} \leqslant\|\Delta v\|_{\sigma}^{2}+c\left\|D^{2} v\right\|_{\omega, Q_{R} \cup Q_{\rho}}^{2} .
$$

Выбирая $\varepsilon>0$ столь малое, что $(1-\varepsilon)^{m}>K^{2}$, из (35) получаем (34).

ДоКАЗАТЕЛЬСтво теоРЕмЫ 1 при $t=0$. Оценка (22), достаточная для доказательства теоремы 1 при $F=\varnothing$, следует из (32) аналогично доказательству леммы 3 для $a, b$ таких, что $a_{*}<b<a<\min \left\{a^{*}, n-2\right\}$. На основе (33) она устанавливается для $a, b$ таких, что $\max \left\{a_{*}, 2-n\right\}<b<a<a^{*}$. При $n=2$ по последнему утверждению леммы 7 она выполнена также при $-\varepsilon<b<a<\varepsilon$.

Рассуждая аналогично доказательству теоремы 1 для $t>0$, при $n>2$ из несправедливости утверждения 1) теоремы 1 получаем оценку (28) с $b=0$, так как $\max \left\{a_{*}, 2-n\right\}<0=b<a<a^{*}$. Теперь, полагая $a=0$, получаем (28) с произвольным $b>a_{*}$, так как $a_{*}<b<a=0<\min \left\{a^{*}, n-2\right\}$.

При $n=2$ из несправедливости утверждения 1$)$ получаем $(28)$ с $b=b_{1} \in(0, \varepsilon)$; полагая $a=b_{1}$, получаем (28) с $b=b_{2} \in(-\varepsilon, 0)$; полагая $a=b_{2}$, получаем (28) с произвольным $b>a_{*}$.

Доказательство теоремы 2 при $t=0$ нуждается в дополнениях по сравнению со случаем $t>0$ только при $a^{*}=n-2, n>2$. В этом случае необходимая оценка (30) леммы 5 выводится из (33) для $a, b$ таких, что $\max \left\{a_{*}, 2-n\right\}<b<a=a^{*}$.

Доказательства теорем 3,4 полностью аналогичны доказательствам теорем 1,2 , поскольку утверждения лемм 1-7 симметричны относительно взаимной замены $R$ и $\rho$. 


\section{§3. Некоторые свойства глобальных решений}

Пусть система (1) задана во всем $\mathbb{R}^{n}$. Рассмотрим решения в $\mathbb{R}^{n} \backslash 0$, т.е. решения, имеющие всего две особые точки 0 и $\infty$. Для такого решения в нуле справедлива альтернатива теоремы 1 и на бесконечности - альтернатива теоремы 3 . Но ситуация не такова, чтобы любая возможность теоремы 1 могла сочетаться с любой возможностью теоремы 3. Фактически если в нуле (или на бесконечности) решение ведет себя как некоторая производная фундаментального решения полилапласиана, то на бесконечности (или в нуле) решение не может вести себя лучше, чем та же производная - в смысле скорости убывания энергии. Более конкретно, справедлива следующая теорема.

Обозначим, как и выше, $U_{R}=\left\|D^{s} u ; L_{2}\left(Q_{R}\right)\right\|^{2}, Q_{R}=\{x: R<|x|<2 R\}$, $F=\left\{a_{j}: j=0,1, \ldots, t-1\right\}, a_{j}=n-2 t+2 j, t=\max _{i} t_{i}$.

Нетривиальньм назовем такое решение системы (1), которое не является полиномом степени меньше $s$.

ТЕОРема 5. Пусть $и \in H_{\mathrm{loc}}^{s}\left(\mathbb{R}^{n} \backslash 0\right)$ - нетривиальное решение системы (1) в $\mathbb{R}^{n} \backslash 0$, и пусть $a_{j} \in F \cap\left(a_{*}, a^{*}\right)$. Тогда справедливь следующие утвержсдения:

i) если при $R \rightarrow 0$ или $R \rightarrow \infty$

$$
c_{1} \leqslant R^{a_{j}} U_{R} \leqslant c_{2}
$$

(решение удовлетворяет утверждению 2) теоремъ 1 или теоремы 3), mo

$$
\inf _{R \in(0, \infty)} R^{a_{j}} U_{R} \geqslant c c_{1}
$$

ii) если при $R \rightarrow 0($ или при $R \rightarrow \infty)$

$$
R^{a_{j}} U_{R} \leqslant c_{2}
$$

то при $R \rightarrow \infty($ или соответственно при $R \rightarrow 0)$

$$
R^{a_{j}} U_{R} \geqslant c_{3}>0
$$

iii) если при $R \rightarrow 0$ и при $R \rightarrow \infty$

$$
R^{a_{j}} U_{R} \leqslant c_{2}
$$

mo

$$
\inf _{R \in(0, \infty)} R^{a_{j}} U_{R} \geqslant c \sup _{R \in(0, \infty)} R^{a_{j}} U_{R}
$$

В случаях i), iii) константа с $>0$ зависит только от $n, s_{i}, t_{i}, K_{i}$.

ДокАЗАтЕльство. Выберем числа $a, b$ так, что $a_{*}<b<a<a^{*},(b, a) \cap F=$ $\left\{a_{j}\right\}, a, b \notin F$, и применим леммы 4,1 .

Если предположение і) выполнено при $R \rightarrow 0$, то из $(24)$ с учетом $a>a_{j}$ при $R \rightarrow 0$ получаем

$$
\left\|D^{s} u\right\|_{\omega, B_{2 \rho}}^{2} \leqslant c\left(\frac{\rho}{\tau}\right)^{a_{j}} U_{\rho}
$$


$0<\tau<\rho<\infty$. Учитывая, что $\omega \asymp 1$ в $Q_{\tau}$, находим

$$
\tau^{a_{j}} U_{\tau} \leqslant c \rho^{a_{j}} U_{\rho}
$$

При $\tau \rightarrow 0$ отсюда следует утверждение і).

Если предположение і) выполнено при $R \rightarrow \infty$, доказательство аналогично с использованием (23) вместо (24).

Если предположение іi) выполнено при $R \rightarrow 0$, то, как и в случае i), получаем (37). Поскольку решение нетривиально, левая часть положительна при некотором $\rho<\infty$. Произвольно фиксируя $\tau$ (например, полагая $\tau=1$ ), при $\rho \rightarrow \infty$ получаем утверждение іi).

Если предположение іi) выполнено при $R \rightarrow \infty$, доказательство аналогично.

Если выполнено предположение іii), то, как и в случае i), из (24) получаем

$$
\tau^{a_{j}} U_{\tau} \leqslant c \rho^{a_{j}} U_{\rho}
$$

$0<\tau<\rho<\infty$. Из (23) при $\rho \rightarrow \infty$ с учетом $b<a_{j}$ аналогично получаем

$$
\tau^{a_{j}} U_{\tau} \leqslant c R^{a_{j}} U_{R}
$$

$0<R<\tau<\infty$. Следовательно, для произвольных $\tau, \rho \in(0, \infty)$ выполнено (38), что доказывает iii).

Отметим, что предположения теоремы 5 во избежание громоздкости сформулированы не в самой слабой форме, а в форме, которую можно ослабить очевидньм образом с апелляцией к теоремам $1-4$. Так, условие $R^{a_{j}} U_{R} \leqslant c$ при $R \rightarrow 0$ в теореме 5 согласно теоремам 1, 2 немедленно следует из более слабого

$$
\begin{aligned}
& \liminf _{R \rightarrow 0} R^{\min \left\{2+a_{j}, a^{*}\right\}} U_{R}=0 \text { при } 2+a_{j} \neq a^{*}, \\
& \liminf _{R \rightarrow 0} R^{a^{*}}\left(\ln \frac{1}{R}\right)^{2} U_{R}=0 \text { при } 2+a_{j}=a^{*} .
\end{aligned}
$$

Случаи 1) и 3) теорем 1 и 3 взаимодействуют следующим образом.

Теорема 6. Пусть $u \in H_{\mathrm{loc}}^{s}\left(\mathbb{R}^{n} \backslash 0\right)$ - нетривиальное решение системь (1) в $\mathbb{R}^{n} \backslash 0$. Если $R^{a} U_{R} \rightarrow 0$ при $R \rightarrow 0$ при любом $a>a_{*}$, то при $R \rightarrow \infty$

$$
R^{a_{*}} U_{R} \geqslant \begin{cases}c, & a_{*} \notin F \\ c(\ln R)^{-2}, & a_{*} \in F\end{cases}
$$

Если $R^{a} U_{R} \rightarrow 0$ при $R \rightarrow \infty$ при любом $a<a^{*}$, то при $R \rightarrow 0$

$$
R^{a^{*}} U_{R} \geqslant \begin{cases}c, & a^{*} \notin F, \\ c\left(\ln \frac{1}{R}\right)^{-2}, & a^{*} \in F .\end{cases}
$$


ДОКАЗАТЕЛЬСТво. Пусть $R^{a} U_{R} \rightarrow 0$ при $R \rightarrow 0$ при любом $a>a_{*}$, и пусть $a_{*} \notin F$. Выберем числа $a, b$ так, что $a_{*}=b<a<a^{*}$ и $a, b$ принадлежат одной компоненте связности множества $\mathbb{R} \backslash F$. По леммам 5,1 получаем

$$
\left\|\left(\frac{r}{\tau}\right)^{a / 2} D^{s} u\right\|_{Q_{R \tau}}^{2} \leqslant c\left(\frac{R}{\tau}\right)^{a} U_{R}+c\left(\frac{\rho}{\tau}\right)^{b} U_{\rho}
$$

$0<R<\tau<\rho<\infty$. При $R \rightarrow 0$ с учетом $a>a_{*}$ отсюда следует

$$
\left\|\left(\frac{r}{\tau}\right)^{a / 2} D^{s} u\right\|_{B_{\tau}}^{2} \leqslant c\left(\frac{\rho}{\tau}\right)^{b} U_{\rho} .
$$

Поскольку решение нетривиально, левая часть положительна при некотором $\tau<\infty$, что дает для больших $\rho$ искомую оценку

$$
\rho^{b} U_{\rho} \geqslant \text { const }>0
$$

Если $a_{*} \in F$, по леммам 6,1 получаем

$$
\left\|\left(\frac{r}{\tau}\right)^{a / 2} D^{s} u\right\|_{Q_{R \tau}}^{2} \leqslant c\left(\frac{R}{\tau}\right)^{a} U_{R}+c\left(1+\ln \frac{\rho}{\tau}\right)^{2}\left(\frac{\rho}{\tau}\right)^{b} U_{\rho}
$$

откуда, аналогично случаю $a_{*} \notin F$, устремляя $R$ к нулю и выбирая $\tau$ достаточно большое, получаем искомую оценку при больших $\rho$.

Вторая часть теоремы доказывается полностью аналогично.

Из теорем вытекает следующий результат о несуммируемости нетривиального решения во всем $\mathbb{R}^{n}$ со степенньм или бистепенньм весом.

СЛЕДСТвИЕ 2. Пусть $u \in H_{\mathrm{loc}}^{s}\left(\mathbb{R}^{n} \backslash 0\right)$ - нетривиальное решение системы (1) в $\mathbb{R}^{n} \backslash 0$. Если $a \in\left(a_{*}, a^{*}\right)$, то

$$
\left\|D^{s} u ; L_{2}\left(\mathbb{R}^{n} ;|x|^{a}\right)\right\|=\infty ;
$$

при $a^{*} \notin F$ или $a_{*} \notin F$ можно допускать также $a=a^{*}$ или $a=a_{*}$. Более того, пусть $a, b, b<a$, такие, что $(b, a) \subset\left(a_{*}, a^{*}\right)$ и интервал $(b, a)$ не содержит внутри точек из $F$ (также потребуем, чтобы $a<a^{*}$ при $a^{*} \in F$ $\left.u b>a_{*} n р и a_{*} \in F\right)$. Тогдa

$$
\left\|D^{s} u ; L_{2}\left(\mathbb{R}^{n} ; \min \left\{|x|^{a} ;|x|^{b}\right\}\right)\right\|=\infty .
$$


ДокаЗАТЕльство. Рассмотрим только бистепенной вес, частным случаем которого является степенной. Пусть $l \in\{0,1, \ldots, t-1\}$ такое, что $a_{l} \leqslant b<a \leqslant a_{l+1}$, где $a_{l}=n-2 t+2 l$. Тогда для того, чтобы интеграл от $\left|D^{s} u\right|^{2}$ с весом $|x|^{a}$ сходился в нуле, по теореме 1 необходимо, чтобы для решения имел место случай 2$)$ c $j \leqslant l$ или случай 3). А чтобы он сходился на бесконечности с весом $|x|^{b}$, по теореме 3 необходимо, чтобы для решения имел место случай 2 ) с $j \geqslant l+1$ или случай 1 ). По теореме $5, \mathrm{i})$ и теореме 6 такие варианты в теореме 1 несовместимы с такими вариантами в теореме 3 , что доказьвает несуммируемость решения по всему $\mathbb{R}^{n}$ с весом $\min \left\{|x|^{a} ;|x|^{b}\right\}$.

Случай $b<a \leqslant a_{0}$ и случай $\left(a_{*}, a^{*}\right) \cap F=\varnothing$ рассматриваются аналогично.

Рассмотрим решения, имеюшие всего одну особую точку -0 или $\infty$. Здесь, в отличие от решений с двумя особыми точками, случай $a^{*} \in F$ или $a_{*} \in F$ не ухудшает оценку.

Назовем бесконечность неособой точкой решения $u$ системы $(1)$ в области $\Omega$ с ограниченным дополнением (в частности, в области $\mathbb{R}^{n} \backslash 0$ ), если $D^{s} u \in L_{2}$ в окрестности $\infty$ и интегральное тождество справедливо с пробными функциями $v$ такими, что $D^{t} v \in L_{2}(\Omega)$ и $v=0$ вблизи $\partial \Omega$.

Теорема 7. Если и - нетривиальное решение системы (1) в $\mathbb{R}^{n} \cup \infty \backslash 0$, $D^{s} u \in L_{2, \text { loc }}\left(\mathbb{R}^{n} \cup \infty \backslash 0\right)$, mo при $R \rightarrow 0$

$$
R^{a^{*}} U_{R} \geqslant c>0
$$

Если $и \in H_{\mathrm{loc}}^{s}\left(\mathbb{R}^{n}\right)$ - нетривиальное решение системы (1) в $\mathbb{R}^{n}$, то при $R \rightarrow \infty$

$$
R^{a_{*}} U_{R} \geqslant c>0
$$

Вторая часть теоремы может быть переформулирована в виде следующего утверждения типа теоремы Лиувилля:

Если $u \in H_{\mathrm{loc}}^{s}\left(\mathbb{R}^{n}\right)$ - решение системь $(1)$ в $\mathbb{R}^{n}$ такое, что

$$
\liminf _{R \rightarrow \infty} R^{a_{*}} U_{R}=0
$$

то $и$ - полином степени $\operatorname{deg} u^{i}<s_{i}$.

ДоказАтЕльство. Докажем первую часть теоремы. Будем рассуждать аналогично доказательству леммы 1. Введем срезающую функцию $\varphi \in C^{\infty}: 0 \leqslant$ $\varphi \leqslant 1, \varphi=1$ при $|x|>2 R, \varphi=0$ при $|x|<R,\left|D^{j} \varphi\right| \leqslant c_{j} R^{-j}$. Положим $v^{i}=K_{i}^{2} \Delta^{-m_{i}} \operatorname{div}^{s_{i}}\left(\omega \varphi D^{s_{i}} u^{i}\right), \omega(r)=\left((r / \tau)^{-a}+(r / \tau)^{-b}\right)^{-1}, a_{*} \leqslant b<a \leqslant a^{*}$, $r=|x|, \tau>R$-параметр. Поскольку $\infty$ - неособая точка решения $u$, в интегральное тождество можно подставить пробную функцию $v_{0}=(v-P) \varphi$, полином $P$ степени $\operatorname{deg} P^{i}<t_{i}$ определим условиями

$$
\int_{Q_{R}} D^{\gamma}\left(v^{i}-P^{i}\right) d x=0, \quad|\gamma|<t_{i}
$$


Следуя доказательству леммы 1 , вместо (7) получаем

$$
\begin{gathered}
\mu(a)\left\|\left(\frac{r}{\tau}\right)^{-a / 2} \omega \varphi D^{s} u\right\|^{2}+\mu(b)\left\|\left(\frac{r}{\tau}\right)^{-b / 2} \omega \varphi D^{s} u\right\|^{2} \\
\leqslant c\left(\left\|D^{s} u\right\|\left(\left\|D^{t} v\right\|+\|D w\|\right)\right)_{Q_{R}},
\end{gathered}
$$

поскольку младшие члены, связанные с производными $\varphi$, распространены только по одному кольцу $Q_{R}$ (в отличие от леммы 1 кольцо $Q_{\rho}$ здесь отсутствует) и могут быть оценены по неравенству Пуанкаре.

При $a, b \in\left(a_{*}, a^{*}\right)$ левая часть (40) оценивает норму $\left\|\varphi D^{s} u\right\|_{\omega}^{2}$. Применяя в правой части неравенство (8) с $q=-a$, находим

$$
\left\|\varphi D^{s} u\right\|_{\omega}^{2} \leqslant \omega(R)^{1 / 2} U_{R}^{1 / 2}\left\|\left(\frac{r}{\tau}\right)^{-a / 2} \omega \varphi D^{s} u\right\|,
$$

откуда с учетом $(r / \tau)^{-a} \omega(r) \leqslant 1$ по неравенству Юнга следует

$$
\left\|\varphi D^{s} u\right\|_{\omega}^{2} \leqslant c \omega(R) U_{R}
$$

Положим теперь $a=a^{*}$. Выберем $q \in\left(-n,-a^{*}\right)$ такое, что $q+2 a^{*}>a_{*}$, и выберем $b \in\left(a_{*}, a^{*}\right)$ такое, что $2 b+q>a_{*}$. Из (40), используя (8), находим

$$
\left\|\left(\frac{r}{\tau}\right)^{-b / 2} \omega \varphi D^{s} u\right\|^{2} \leqslant c\left(\frac{R}{\tau}\right)^{-q / 2} U_{R}^{1 / 2}\left\|\varphi D^{s} u\right\|_{\sigma}
$$

$\sigma(r)=(r / \tau)^{q} \omega^{2}$. Поскольку $\sigma(r) \asymp\left((r / \tau)^{-2 a-q}+(r / \tau)^{-2 b-q}\right)^{-1}$, где $2 a+q$, $2 b+q \in\left(a_{*}, a^{*}\right)$, соответствуюшую норму можно оценить по (41). Это дает

$$
\left\|\left(\frac{r}{\tau}\right)^{-b / 2} \omega \varphi D^{s} u\right\|^{2} \leqslant c\left(\frac{R}{\tau}\right)^{a} U_{R}
$$

Если допустить, что (39) не вьполнено, т.е.

$$
\liminf _{R \rightarrow 0} R^{a^{*}} U_{R}=0
$$

то отсюда при $R \rightarrow 0$ следует $D^{s} u=0$ во всем $\mathbb{R}^{n}$, что противоречит предположению о нетривиальности решения $u$. Первая часть теоремы доказана.

Вторая часть теоремы доказывается полностью аналогично.

\section{§4. О точности оценки интервала правильного поведения решений}

Для уравнений и систем второго порядка установим точность оценки интервала $\left(a_{*}, a^{*}\right)$ правильного поведения решений, которая дается функцией $M_{s, t}$. А именно построим примеры уравнений и систем второго порядка, имеюших решения такие, что

$$
c_{1} \leqslant R^{b} U_{R} \leqslant c_{2}
$$

при $R \in(0, \infty)$, где $b=b^{*}$ или $b=b_{*}$. Отметим, что первый пример такого рода в теории регулярности решений построен в $[7 ;$ гл. 6$]$. 
1. Дивергентные уравнения на плоскости: $s=t=1, n=2, N=1$, $K \in(0,1)$. Рассмотрим уравнение

$$
\Delta u+\left(\gamma^{2}-1\right) D_{i}\left(\frac{x_{i} x_{j}}{|x|^{2}} D_{j} u\right)=0
$$

$\gamma>1, i, j=1,2$, по повторяюшимся индексам идет суммирование. Оно имеет решение $u(x)=x_{1}|x|^{\alpha}, \alpha=-1 \pm 1 / \gamma$. Для дивергентных уравнений и систем с симметрической матрицей коэффициентов $K=(\lambda-1) /(\lambda+1)$ [7; с. 34], где $\lambda-$ модуль эллиптичности (отношение наибольшего собственного числа матрицы коэффициентов к наименьшему). В данном случае, очевидно, $\lambda=\gamma^{2}$. По формуле (5) при $n=2, m=1$ находим

$$
b^{*}=-b_{*}=2\left(\frac{1-K}{1+K}\right)^{1 / 2}=\frac{2}{\gamma}
$$

Следовательно, при $\alpha=-1-1 / \gamma$ решение $u(x)=x_{1}|x|^{\alpha}$ удовлетворяет (42) с $b=b^{*}$, а при $\alpha=-1+1 / \gamma-\mathrm{c} b=b_{*}$.

2. Дивергентные системы: $s=t=1, N=n \geqslant 2, K \in(0,1)$ при $n \leqslant 8$, $0<K \leqslant(\sqrt{n-1}+\sqrt{2 n})\left((\sqrt{2 n}-\sqrt{n-1})^{2}+(n-2)^{2}\right)^{-1 / 2}$ при $n>8$. Рассмотрим систему

$$
\Delta u^{i}+D_{k}\left(b_{i k} b_{j l} D_{l} u^{j}\right)=0
$$

$b_{i k}=c \delta_{i k}+d x_{i} x_{k}|x|^{-2}, i, j, k, l=1, \ldots, n, c, d$ - константы, $\delta_{i k}-$ символ Кронекера. При $c=n-2, d=n$ это - система де Джорджи, различные модификации которой рассматривались многими авторами. Например, в $[7$; гл. 6$]$ при соответствующем выборе $c, d$ установлена точность условия $K<\left(1+(n-2)^{2} /(n-1)\right)^{-1 / 2}$ для гёльдеровости обобшенных решений дивергентных систем второго порядка. Непосредственными вычислениями проверяется, что данная система имеет решение

$$
u(x)=x|x|^{\alpha}, \quad \alpha=\alpha_{ \pm}=-\frac{n}{2} \pm\left(\frac{n^{2}}{4}-(n-1) d \frac{n c+d}{1+(c+d)^{2}}\right)^{1 / 2} .
$$

Матрица коэффициентов системы симметрическая как $(n N \times n N)$-матрица (в том смысле, что $A_{k l}^{i j}=A_{l k}^{j i}$, где $\left.A_{k l}^{i j}=\delta_{i j} \delta_{k l}+b_{i k} b_{j l}\right)$, поэтому $K=(\lambda-1) /(\lambda+1)$, как и в предыдущем случае. При интерпретации $\left(A_{k l}^{i j}\right)$ как квадратной матрицы, действуюшей на векторы $\xi_{l}^{j}$, легко видеть, что наименњшее собственное число единица $\left(\xi_{l}^{j}\right.$ ортогонально $\left.b_{j l}\right)$, наибольшее собственное число $\lambda=1+(n-1) c^{2}+$ $(c+d)^{2}\left(\xi_{l}^{j}\right.$ параллельно $\left.b_{j l}\right)$. Введем параметризацию $c+d=\rho \cos \varphi, c(n-1)^{1 / 2}=$ $\rho \sin \varphi$. Тогда $K=\left(1+2 \rho^{-2}\right)^{-1}$ не зависит от $\varphi$. Исследуя выражение для $\alpha$ на экстремум по $\varphi$ с помощью дифференцирования, получаем, что $\alpha_{+}$максимально, а $\alpha_{-}$минимально при

$$
\cos 2 \varphi=n^{-2}\left(2(n-1)^{1 / 2}\left(n^{2}-(n-2)^{2} K^{2}\right)^{1 / 2}-(n-2)^{2} K\right), \quad \sin 2 \varphi>0 .
$$


Непосредственными вычислениями проверяется, что в этом случае

$$
\begin{aligned}
\alpha_{ \pm} & =-\frac{n}{2} \pm\left(\frac{n^{2}}{4}-\frac{2(n-1)}{\cos 2 \varphi+1+\rho^{-2}}\left(\cos 2 \varphi+\frac{n-2}{2(n-1)^{1 / 2}} \sin 2 \varphi\right)\right)^{1 / 2} \\
& =-\frac{n}{2} \pm \frac{1}{2\left(1-K^{2}\right)^{1 / 2}}\left(\left(n^{2}-(n-2)^{2} K^{2}\right)^{1 / 2}-2 K(n-1)^{1 / 2}\right) .
\end{aligned}
$$

По формуле (5) это равно $-\left(n+b^{*}\right) / 2$ в случае знака минус и $-\left(n+b_{*}\right) / 2$ в случае знака плюс, если в (5) минимум достигается при $j=1$. Последнее происходит при $K \in(0,1), n \leqslant 8$ и при $0<K \leqslant(\sqrt{2 n}+\sqrt{n-1})\left((\sqrt{2 n}-\sqrt{n-1})^{2}+(n-2)^{2}\right)^{-1 / 2}$, $n>8, m=1$. При таких значениях $K(42)$ выполнено с $b=b^{*}$ при $\alpha=\alpha_{-}$и с $b=b_{*}$ при $\alpha=\alpha_{+}$.

3. Уравнения типа Кордеса: $s=2, t=0, N=1, n \geqslant 2, K \in(0,1)$. В данном случае устанавливается точность только для показателя $b^{*}$. Рассмотрим уравнение

$$
\Delta u+(\gamma-1) \frac{x_{i} x_{j}}{|x|^{2}} D_{i} D_{j} u=0
$$

$\gamma>1, i, j=1, \ldots, n$. Оно имеет решение $u(x)=|x|^{\alpha}, \alpha=1-(n-1) / \gamma$. Для недивергентных уравнений, как показано вьшше,

$$
K^{2}=n-\frac{\left(A_{i i}\right)^{2}}{A_{i j}^{2}}=(n-1) \frac{(\gamma-1)^{2}}{n-1+\gamma^{2}},
$$

где $A_{i j}=\delta_{i j}+(\gamma-1) x_{i} x_{j}|x|^{-2}$ - коэффициенты уравнения. Имеем $K \in(0,1)$ при $\gamma \in(1,2(n-1) /(n-2))$. Подставляя полученное значение $K$ в формулу $(6)$ с $m=1$, находим $b^{*}=2-n+2(n-1) / \gamma$. Следовательно, $u(x)=|x|^{\alpha}$ удовлетворяет (42) c $b=b^{*}$.

4. Системы типа Кордеса: $s=2, t=0, N=n^{2}, K \in(0,1)$ при $n=2$, $0<K<((2+\sqrt{3}) / 4)^{1 / 2}$ при $n>2$. В данном случае устанавливается точность только для показателя $b_{*}$. Рассмотрим вектор-функцию $u$ с компонентами

$$
u^{i j}(x)=\left(x_{i} x_{j}-\frac{1}{n} \delta_{i j}|x|^{2}\right)|x|^{\alpha},
$$

$i, j=1, \ldots, n, \alpha \in(-n / 2,0)$. Для нее справедливо равенство

$$
\frac{\left|D^{2} u(x)\right|^{2}}{|\Delta u(x)|^{2}}=M_{2}\left(b, \lambda_{2}\right)
$$

c $b=-n-2 \alpha$. Действительно, дифференцирование дает

$$
\begin{aligned}
|x|^{-\alpha} D_{k} D_{l} u^{i j}(x)= & \delta_{i k} \delta_{j l}+\delta_{i l} \delta_{j k}-\frac{2+\alpha}{n} \delta_{i j} \delta_{k l} \\
& +\alpha|x|^{-2}\left(\delta_{i k} x_{j} x_{l}+\delta_{j k} x_{i} x_{l}+\delta_{i l} x_{j} x_{k}+\delta_{j l} x_{i} x_{k}\right. \\
& \left.+\delta_{k l} x_{i} x_{j}-\frac{2+\alpha}{n} \delta_{i j} x_{k} x_{l}\right)+\alpha(\alpha-2) x_{i} x_{j} x_{k} x_{l}|x|^{-4} \\
\Delta u^{i j}= & \alpha(\alpha+n+2)|x|^{-2} u^{i j}
\end{aligned}
$$


Непосредственно вычисляя, получаем

$$
\begin{aligned}
\left|D^{2} u\right|^{2}= & \sum_{i, j, k, l=1}^{n}\left(D_{k} D_{l} u^{i j}\right)^{2}=\left(\alpha^{2}(\alpha+n+2)^{2}+(n+2 \alpha)(2 n(2 \alpha+3)\right. \\
& \left.\left.-(n-1)(\alpha+2)^{2}\right)\right) \frac{n-1}{n}|x|^{2 \alpha}, \\
|\Delta u|^{2}= & \alpha^{2}(\alpha+n+2)^{2} \frac{n-1}{n}|x|^{2 \alpha},
\end{aligned}
$$

откуда следует указанное равенство с $b=-n-2 \alpha$.

Равенство $M_{2}(b)=M_{2}\left(b, \lambda_{2}\right)$ имеет место при $-n<b \leqslant \min \{0 ; 1+\sqrt{3}-n\}$ (для $n \geqslant 3$ на несколько более широком интервале, но правый конец интервала не выражается в радикалах). Построим недивергентную систему, удовлетворяюшую (2) с $K^{2}=1 / M_{2}(b), b=-n-2 \alpha$, решением которой является функция $u$. Такой будет, например, система

$$
\Delta u^{i j}-\sum_{k, l, p, q=1}^{n} B_{k l}^{i j p q}(x) D_{k} D_{l} u^{p q}=0
$$

где $B_{k l}^{i j p q}(x)=\Delta u^{i j}(x)\left|D^{2} u(x)\right|^{-2} D_{k} D_{l} u^{p q}(x)$, а также система

$$
\Delta u^{i j}-B^{i j}(x)\left|D^{2} u\right|=0,
$$

где $B^{i j}(x)=\Delta u^{i j}(x) /\left|D^{2} u(x)\right|$. Очевидно, произвольная функция $u=\left(u^{i j}\right)$ удовлетворяет каждому из этих равенств. Что касается величины $K$, в первом случае по неравенству Коши

$$
K^{2} \leqslant \sup _{x} \sum_{i, j, k, l, p, q=1}^{n}\left(B_{k l}^{i j p q}(x)\right)^{2}=\sup _{x} \frac{|\Delta u(x)|^{2}}{\left|D^{2} u(x)\right|^{2}}=\frac{1}{M_{2}(b)} .
$$

Во втором случае оценка аналогична. Следовательно, для этих систем $b_{*} \leqslant-n-2 \alpha$. Функция $u$ удовлетворяет (42) с $b=-n-2 \alpha$, а так как при $b \in\left(b_{*}, 0\right)$ это невозможно по теореме 1 или теореме 3 , имеем $b_{*}=-n-2 \alpha$.

5. Вполне дивергентные уравнения: $s=0, t=2, N=1, n \geqslant 2, K \in(0,1)$. В этом случае устанавливается точность только для показателя $b_{*}$. Рассмотрим уравнение

$$
\Delta u+(\gamma-1) D_{i} D_{j}\left(\frac{x_{i} x_{j}}{|x|^{2}} u\right)=0
$$

$\gamma>1, i, j=1, \ldots, n$. Оно имеет решение $u(x)=|x|^{\alpha}, \alpha=-(1-1 / \gamma)(n-1)$. Как и для уравнений типа Кордеса, имеем

$$
K^{2}=n-\frac{\left(A_{i i}\right)^{2}}{A_{i j}^{2}}=(n-1) \frac{(\gamma-1)^{2}}{n-1+\gamma^{2}},
$$

где $A_{i j}=\delta_{i j}+(\gamma-1) x_{i} x_{j}|x|^{-2}$ - коэффициенты уравнения. Если $\gamma \in(1,2(n-$ $1) /(n-2))$, то $K \in(0,1)$. Подставляя полученное значение $K$ в формулу (6) с $m=1$, находим $b_{*}=n-2-2(n-1) / \gamma$. Следовательно, решение $u(x)=|x|^{\alpha}$ удовлетворяет (42) с $b=b_{*}$. 


\section{Список литературы}

1. Kondrat'ev V.A., Oleinik O. A. On the behaviour at infinity of solutions of elliptic systems with a finite energy integral // Arch. Ration. Mech. Anal. 1987. V. 93. № 1. P. 75-99.

2. Serrin J. Local behaviour of solutions of quasilinear elliptic equations // Acta Math. 1964. V. 111. P. 247-302.

3. Cordes H. O. Über die erste Randvertaufgabe bei quasilinear Differentialgleichungen zweiter Ordnung in mehr als zwei Variablen // Math. Ann. 1956. V. 131. № 3. P. 287-312.

4. Калита $E$. A. Об особых точках решений нелинейных эллиптических уравнений и систем высокого порядка // Матем. сб. 1993. Т. 184. № 7. С. 117-143.

5. Калита E. A. Теорема Лиувилля для эллиптических систем типа Кордеса высокого порядка // Укр. матем. журн. 1991. Т. 43. № 2. С. 199-205.

6. Kaлита E. A. Разрешимость нелинейных эллиптических систем в пространствах слабее естественного энергетического // Изв. РАН. Сер. матем. 1997. Т. 61. № 2. С. 53-80.

7. Koshelev A.I., Chelkak S. I. Regularity of solutions of quasilinear elliptic systems. Leipzig: Teubner, 1985.

8. Берг Й., Лёфстрём Й. Интерполяционные пространства. Введение. М.: Мир, 1980.

9. Калита E. A. Регулярность по Морри решений нелинейных эллиптических систем произвольного порядка при ограничениях на модуль эллиптичности // Укр. матем. журн. 2002. Т. 54. № 11. С. 1452-1466.

10. Дынькин Е. М., Осиленкер Б. П. Весовые оценки сингулярных интегралов и их приложения // Итоги науки и техники. Матем. анализ. Т. 21. М.: ВИНИТИ, 1983. С. 42-129.

Институт прикладной математики и механики

Поступила в редакцию

НАН Украины, г. Донецк

06.06 .2003

E-mail: kalita@iamm.ac.donetsk.ua 